\title{
Nonhyperbolic Periodic Orbits of Vector Fields in the Plane Revisited
}

\author{
Denis de Carvalho Braga, ${ }^{1}$ Luis Fernando Mello, ${ }^{2}$ and Antonio Carlos Zambroni de Souza ${ }^{3}$ \\ ${ }^{1}$ Campus Avançado de Itabira, Universidade Federal de Itajubá, Rua São Paulo 377, Bairro Amazonas, 35.900-373 Itabira, MG, Brazil \\ ${ }^{2}$ Instituto de Matemática e Computação, Universidade Federal de Itajubá, Avenida BPS 1303, Pinheirinho, 37.500-903 Itajubá, \\ MG, Brazil \\ ${ }^{3}$ Instituto de Sistemas Elétricos e Energia, Universidade Federal de Itajubá, Avenida BPS 1303, Pinheirinho, 37.500-903 Itajubá, \\ MG, Brazil
}

Correspondence should be addressed to Luis Fernando Mello; lfmelo@unifei.edu.br

Received 23 June 2013; Accepted 8 July 2013

Academic Editor: Sakthivel Rathinasamy

Copyright (C) 2013 Denis de Carvalho Braga et al. This is an open access article distributed under the Creative Commons Attribution License, which permits unrestricted use, distribution, and reproduction in any medium, provided the original work is properly cited.

The main goal of this paper is to present a theory of approximation of periodic orbits of vector fields in the plane. From the theory developed here, it is possible to obtain an approximation to the curve of nonhyperbolic periodic orbits in the bifurcation diagram of a family of differential equations that has a transversal Hopf point of codimension two. Applications of the developed theory are made in Liénard-type equations and in Bazykin's predator-prey system.

\section{Introduction}

The existence of a curve of nonhyperbolic periodic orbits in the bifurcation diagram of a family of differential equations that has a transversal Hopf point of codimension two can be demonstrated with the theories presented in $[1,2]$. However, these theories do not allow us to find or even approximate the curve of nonhyperbolic periodic orbits, except in very special cases as in [3]. On the other hand, good approximations to this curve are essential not only to mathematicians, but primarily for engineers, physicists, and other users of mathematics.

In general, the curve of nonhyperbolic periodic orbits is obtained by numerical methods as in [4] or through specific softwares such as [5], for instance. An analytical alternative proposed in this paper is to generalize the theory of approximation of periodic orbits of [6], using some results and notations of $[1,2]$, in order to obtain an approximation to the curve of nonhyperbolic periodic orbits of a family of differential equations that has transversal Hopf bifurcations of codimension two. Furthermore, the theory developed here does not need normal forms of the vector field in the neighborhood of the Hopf points.
Article [7], among other cases, treats also the generalized Hopf bifurcation in general as $n$-dimensional systems. In particular, it provides quadratic asymptotics for the bifurcation parameter values corresponding to the nonhyperbolic limit cycle, and for this cycle itself. Moreover, these asymptotics are implemented into the standard software MATCONT [5], allowing to automatically initialize the continuation of the cycle-saddle-node curve from the generalized Hopf point. However, the authors believe that the constructions presented here are independent and self-contained. More precisely, both articles give an approximation to the curve of nonhyperbolic periodic orbits of a family of differential equations that has transversal Hopf bifurcations of codimension two. Here we present this theory for 2-dimensional systems without the use of normal forms while in [7], the authors present $n$ dimensional systems using normal forms.

This paper is organized as follows. In Section 2, the theory of approximation of periodic orbits for vector fields in the plane is developed. The stability of the approximate periodic orbits is discussed in Section 3. In Section 4, applications of the theory in Liénard-type differential equations are made, while applications to the Bazykin's predator-prey system are 
made in Section 5. Concluding comments about the results obtained here are in Section 6.

\section{Approximation of Periodic Orbits}

Consider a family of the differential equations

$$
\mathbf{x}^{\prime}=f(\mathbf{x}, \xi)
$$

where $f: W \times U \rightarrow \mathbb{R}^{2}, W \subset \mathbb{R}^{2}$ is an open set in $\mathbb{R}^{2}$, $f \in \mathscr{C}^{\infty}\left(W \times U, \mathbb{R}^{2}\right)$, and $\xi=(\mu, \nu) \in U \subset \mathbb{R}^{2}$ is the parameter vector. Let $\left(\mathbf{x}_{0}(\xi), \xi\right) \in W \times U$ be an equilibrium point of (1); that is, $f\left(\mathbf{x}_{\mathbf{0}}(\xi), \xi\right)=\mathbf{0}$ for $\xi \in U$. Suppose the following assumption:

(H1) the linear part of the vector field $f: W \times U \rightarrow$ $\mathbb{R}^{2}$, evaluated at $\left(\mathbf{x}_{0}(\xi), \xi\right)$ and denoted by $A(\xi)=$ $D f\left(\mathbf{x}_{\mathbf{0}}(\xi), \xi\right)$, has eigenvalues $\lambda$ and $\bar{\lambda}$, with $\lambda(\xi)=$ $\gamma(\xi)+i \eta(\xi)$. For $\xi_{0}=\left(\mu_{0}, \nu\right) \in U, \gamma\left(\xi_{0}\right)=0, \partial_{\mu} \gamma\left(\xi_{0}\right) \neq$ 0 , and $\eta\left(\xi_{0}\right)=\omega_{0}(\nu)>0$, where

$$
\partial_{\mu} \gamma\left(\xi_{0}\right)=\left.\frac{\partial}{\partial \mu} \gamma(\xi)\right|_{\xi=\xi_{0}}
$$

There is no loss of generality in considering that $\mathbf{x}_{0}(\xi)=\mathbf{0}$ for all $\xi \in U,(0,0) \in U$ and $\mu_{0}=0$. Just make a translation of the equilibrium point and of the critical parameter to their origins and adjust in a convenient way the sets $W \subset \mathbb{R}^{2}$ and $U \subset \mathbb{R}^{2}$. By doing this, (1) can be rewritten as

$$
\mathbf{x}^{\prime}=A(\xi) \mathbf{x}+G(\mathbf{x}, \xi)
$$

where $(\mathbf{x}, \xi) \mapsto G(\mathbf{x}, \xi)$ is a smooth vector field with Taylor expansion around $\mathbf{x}=\mathbf{0}$, starting with second-order terms at least, as follows:

$$
\begin{aligned}
G(\mathbf{x}, \xi)= & \frac{1}{2} B(\mathbf{x}, \mathbf{x}, \xi)+\frac{1}{6} C(\mathbf{x}, \mathbf{x}, \mathbf{x}, \xi)+\frac{1}{24} D(\mathbf{x}, \mathbf{x}, \mathbf{x}, \mathbf{x}, \xi) \\
& +\frac{1}{120} E(\mathbf{x}, \mathbf{x}, \mathbf{x}, \mathbf{x}, \mathbf{x}, \xi)+O_{G}\left(\|\mathbf{x}\|^{6}, \xi\right),
\end{aligned}
$$

where

$$
\begin{gathered}
B_{i}(\mathbf{x}, \mathbf{y}, \xi)=\left.\sum_{j, k=1}^{2} \frac{\partial^{2}}{\partial \eta_{j} \partial \eta_{k}} G_{i}(\eta, \xi)\right|_{\eta=0} x_{j} y_{k}, \\
C_{i}(\mathbf{x}, \mathbf{y}, \mathbf{u}, \xi)=\left.\sum_{j, k, l=1}^{2} \frac{\partial^{3}}{\partial \eta_{j} \partial \eta_{k} \partial \eta_{l}} G_{i}(\eta, \xi)\right|_{\eta=0} x_{j} y_{k} u_{l}, \\
D_{i}(\mathbf{x}, \mathbf{y}, \mathbf{u}, \mathbf{v}, \xi)=\left.\sum_{j, k, l, r=1}^{2} \frac{\partial^{4}}{\partial \eta_{j} \partial \eta_{k} \partial \eta_{l} \partial \eta_{r}} G_{i}(\eta, \xi)\right|_{\eta=0} x_{j} y_{k} u_{l} v_{r}, \\
E_{i}(\mathbf{x}, \mathbf{y}, \mathbf{u}, \mathbf{v}, \mathbf{w}, \xi) \\
=\left.\sum_{j, k, l, r, p=1}^{2} \frac{\partial^{5}}{\partial \eta_{j} \partial \eta_{k} \partial \eta_{l} \partial \eta_{r} \partial \eta_{p}} G_{i}(\eta, \xi)\right|_{\eta=0} x_{j} y_{k} u_{l} v_{r} w_{p}
\end{gathered}
$$

are the components of symmetric multilinear functions $B, C$, $D$, and $E$.

Let $q(\xi) \in \mathbb{C}^{2}$ be an eigenvector corresponding to the eigenvalue $\lambda(\xi)$, and let $p(\xi) \in \mathbb{C}^{2}$ be an adjoint eigenvector corresponding to the eigenvalue $\bar{\lambda}(\xi)$ satisfying

$$
\begin{aligned}
& A(\xi) q(\xi)=\lambda(\xi) q(\xi), \\
& A(\xi)^{T} p(\xi)=\bar{\lambda}(\xi) p(\xi),
\end{aligned}
$$

and the normalization

$$
\langle p(\xi), q(\xi)\rangle=\sum_{i=1}^{2} \bar{p}_{i}(\xi) q_{i}(\xi)=1,
$$

where $\langle\cdot, \cdot\rangle: \mathbb{C}^{2} \times \mathbb{C}^{2} \rightarrow \mathbb{C}$ is the standard inner product in $\mathbb{C}^{2}$ and $A(\xi)^{T}$ is the transpose of the matrix $A(\xi)$. The set $\{q(\xi), \bar{q}(\xi)\}$ is a basis of $\mathbb{C}^{2}$ and the subspace of $\mathbb{C}^{2}$ defined by

$$
\mathbb{R}_{0}^{2}=\left\{(\mathbf{x}, \mathbf{y}) \in \mathbb{C}^{2}: \mathbf{x}, \mathbf{y} \in \mathbb{R}^{2}, \mathbf{y}=\mathbf{0}\right\}
$$

is isomorphic to the vector space $\mathbb{R}^{2}$. Taking into account the isomorphism between $\mathbb{R}^{2}$ and $\mathbb{R}_{0}^{2}$, if $(\mathbf{x}, \mathbf{0}) \in \mathbb{R}_{0}^{2}$, then the notation used is $\mathbf{x} \in \mathbb{R}^{2}$. Thus, every vector $\mathbf{x} \in \mathbb{R}^{2}$ can be uniquely represented as a linear combination of elements of $\{q(\xi), \bar{q}(\xi)\}$; that is, there is $z \in \mathbb{C}$ such that

$$
\mathbf{x}=z q(\xi)+\overline{z q}(\xi) .
$$

It is easy to show that $\langle p(\xi), \bar{q}(\xi)\rangle=0$ and $z=\langle p(\xi), \mathbf{x}\rangle$. So (1) can be written as a complex family of differential equations as follows:

$$
z^{\prime}=g(z, \bar{z}, \xi)
$$

for $\left\|\xi-\xi_{0}\right\|$ sufficiently small, where $g \in \mathscr{C}^{\infty}(\mathbb{C} \times \mathbb{C} \times U, \mathbb{C})$ and

$$
g(z, \bar{z}, \xi)=\lambda(\xi) z+\langle p(\xi), G(z q(\xi)+\overline{z q}(\xi), \xi)\rangle .
$$

The function $(z, \bar{z}, \xi) \mapsto g(z, \bar{z}, \xi)$ has formal Taylor series

$$
g(z, \bar{z}, \xi)=\lambda(\xi) z+\sum_{k=2}^{\infty} \sum_{j=0}^{k} \frac{1}{(k-j) ! j !} g_{k-j, j}(\xi) z^{k-j} \bar{z}^{j},
$$

where

$$
g_{k-j, j}(\xi)=\left.\frac{\partial^{k}}{\partial z^{k-j} \partial \bar{z}^{j}}\langle p(\xi), G(z q(\xi)+\bar{z} \bar{q}(\xi), \xi)\rangle\right|_{z=0},
$$

for $k=2,3, \ldots$ and $j=0, \ldots, k$.

The coefficients $g_{k-j, j}(\xi)$ for $k=2,3, \ldots$ and $j=0, \ldots, k$ play an important role in the method of approximation of a family of periodic orbits of (1). A simple way to calculate these coefficients, alternative to (14), is through the symmetric multilinear functions. From the symmetric bilinear function $(\mathbf{x}, \mathbf{y}, \xi) \mapsto B(\mathbf{x}, \mathbf{y}, \xi)$ and $(10)$, it follows that

$$
\begin{aligned}
B(z q(\xi)+\bar{z} \bar{q}(\xi), z q(\xi)+\overline{z q}(\xi), \xi) \\
=B(q(\xi), q(\xi), \xi) z^{2}+2 B(q(\xi), \bar{q}(\xi), \xi) z \bar{z} \\
\quad+B(\bar{q}(\xi), \bar{q}(\xi), \xi) \bar{z}^{2},
\end{aligned}
$$


and, therefore,

$$
\begin{aligned}
& g_{2,0}(\xi)=\langle p(\xi), B(q(\xi), q(\xi), \xi)\rangle, \\
& g_{1,1}(\xi)=\langle p(\xi), B(q(\xi), \bar{q}(\xi), \xi)\rangle, \\
& g_{0,2}(\xi)=\langle p(\xi), B(\bar{q}(\xi), \bar{q}(\xi), \xi)\rangle .
\end{aligned}
$$

Similarly, for the symmetric trilinear function $(\mathbf{x}, \mathbf{y}, \mathbf{u}, \xi) \mapsto$ $C(\mathbf{x}, \mathbf{y}, \mathbf{u}, \xi)$,

$$
\begin{aligned}
& g_{3,0}(\xi)=\langle p(\xi), C(q(\xi), q(\xi), q(\xi), \xi)\rangle, \\
& g_{2,1}(\xi)=\langle p(\xi), C(q(\xi), q(\xi), \bar{q}(\xi), \xi)\rangle, \\
& g_{1,2}(\xi)=\langle p(\xi), C(q(\xi), \bar{q}(\xi), \bar{q}(\xi), \xi)\rangle, \\
& g_{0,3}(\xi)=\langle p(\xi), C(\bar{q}(\xi), \bar{q}(\xi), \bar{q}(\xi), \xi)\rangle,
\end{aligned}
$$

and so on for other symmetric multilinear functions.

The aim of the theory of approximation of periodic orbits in [6] is to build an approximation for a periodic orbit of the complex differential equation (11), from the solution of the linear differential equation

$$
z^{\prime}=\lambda(\xi) z
$$

for $\xi=\xi_{0}$. This linear differential equation has the solution

$$
z(t)=z_{0} e^{\lambda(\xi) t},
$$

where $z_{0} \in \mathbb{C}$. For $\xi=\xi_{0}$, it follows that

$$
z(t)=z_{0} e^{i \omega_{0}(v) t}
$$

and making the change in time $s=\omega_{0}(\nu) t$, this solution is periodic of period $2 \pi$ in the variable $s$. To formalize the method, consider the functions $(\epsilon, \nu) \mapsto \mu=\phi(\epsilon, \nu),(\epsilon, \nu) \mapsto$ $\omega(\epsilon, \nu)$ and the change of coordinates and time

$$
z(t)=w(s, \epsilon, \nu), \quad s=\omega(\epsilon, \nu) t, \quad \omega\left(\xi_{0}\right)=\omega_{0}(\nu),
$$

where

$$
\epsilon=\frac{1}{2 \pi} \int_{0}^{2 \pi} e^{-i s} w(s, \epsilon, \nu) d s .
$$

Note that the parameter $\epsilon$, as defined in (22), is a complex number or, more precisely, a complex function whose independent variable is $v$. However, it is possible, through a change of variables, to consider the parameter $\epsilon$ as a real number. In fact, as

$$
\epsilon=\varepsilon e^{i \phi}=\frac{1}{2 \pi} \int_{0}^{2 \pi} e^{-i s} w(s, \epsilon, \nu) d s,
$$

it follows that

$$
\begin{aligned}
\varepsilon & =|\epsilon|=\frac{e^{-i \phi}}{2 \pi} \int_{0}^{2 \pi} e^{-i s} w(s, \epsilon, \nu) d s \\
& =\frac{1}{2 \pi} \int_{0}^{2 \pi} e^{-i(s+\phi)} w(s, \epsilon, \nu) d s .
\end{aligned}
$$

Thus, making the change of variable $u=s+\phi$ in (24) and setting $(u, \epsilon, \nu) \mapsto \widetilde{w}(u, \epsilon, \nu)=w(u-\phi, \epsilon, \nu)$,

$$
\begin{aligned}
\varepsilon & =|\epsilon|=\frac{1}{2 \pi} \int_{\phi}^{2 \pi+\phi} e^{-i u} w(u-\phi, \epsilon, \nu) d u \\
& =\frac{1}{2 \pi} \int_{0}^{2 \pi} e^{-i u} \widetilde{w}(u, \epsilon, \nu) d u,
\end{aligned}
$$

since the function $(u, \epsilon, \nu) \mapsto e^{-i u} \widetilde{w}(u, \epsilon, \nu)$ is periodic of period $2 \pi$ in the variable $s$. Therefore, by (25), the parameter $\epsilon$ as defined in (22) will be considered a real parameter.

The generalization of the theory of approximation of periodic orbits introduced in [6] consists in achieving an approximation to the two-parameter family of periodic orbits

$$
\left\{(s, \epsilon, \nu) \in \mathbb{R} \times U_{\epsilon} \longmapsto w(s, \epsilon, \nu) \in \mathbb{C}:(\epsilon, \nu) \in U_{\epsilon}\right\},
$$

where $U_{\epsilon}=\left\{(\epsilon, \nu) \in \mathbb{R}^{2}:(\phi(\epsilon, \nu), \nu) \in U\right\}$.

The change in time $s=\omega(\epsilon, \nu) t$ is essential, since the period of the family of periodic orbits (26) is unknown and, therefore, the change in time is used only to provide an approximation of the known period $2 \pi$ for the family of periodic orbits (26). If $(\epsilon, \nu) \mapsto T(\epsilon, \nu)$ denotes the period of the family of periodic orbits, then

$$
\omega(\epsilon, \nu)=\frac{2 \pi}{T(\epsilon, \nu)} .
$$

In other words, the knowledge of the function $(\epsilon, \nu) \mapsto \omega(\epsilon, \nu)$ completely determines the period of the family of periodic orbits of (26).

By changing the coordinates and time (21) and applying the chain rule, the complex differential equation (11) is rewritten as

$$
\omega(\epsilon, \nu) \frac{d}{d s} w(s, \epsilon, \nu)=g(w(s, \epsilon, \nu), \bar{w}(s, \epsilon, \nu), \phi(\epsilon, \nu), \nu) .
$$

Approximations to the functions $(s, \epsilon, \nu) \mapsto w(s, \epsilon, \nu)$, $(\epsilon, \nu) \mapsto \mu=\phi(\epsilon, \nu)$ and $(\epsilon, \nu) \mapsto \omega(\epsilon, \nu)$ are obtained through (28) and the formal power series

$$
\left(\begin{array}{c}
w(s, \epsilon, \nu) \\
\phi(\epsilon, \nu) \\
\omega(\epsilon, \nu)-\omega_{0}(\nu)
\end{array}\right)=\sum_{k=1}^{\infty} \frac{1}{k !}\left(\begin{array}{c}
w_{k}(s, \nu) \\
\mu_{k}(\nu) \\
\omega_{k}(\nu)
\end{array}\right) \epsilon^{k} .
$$

A property of the terms of the sequence $\left\{w_{k}(s, \nu)\right\}_{k \in \mathbb{N}}$, widely used in this theory of approximation of periodic orbits of vector fields in $\mathbb{R}^{2}$, is obtained in Proposition 1 .

Proposition 1. Each term of the sequence $\left\{w_{k}(s, \nu)\right\}_{k \in \mathbb{N}}$ satisfies

$$
\left[w_{k}\right]=\frac{1}{2 \pi} \int_{0}^{2 \pi} e^{-i s} w_{k}(s, v) d s= \begin{cases}1, & k=1 \\ 0, & k=2,3, \ldots\end{cases}
$$


Proof. Setting $\mathscr{W}=\left\{w: \mathbb{R} \times U_{\epsilon} \rightarrow \mathbb{C}: w \in \mathscr{C}^{\infty}\left(\mathbb{R} \times U_{\epsilon}, \mathbb{C}\right)\right\}$, the proof is an immediate consequence of the definition of linear map

$$
\begin{aligned}
& {[\quad]: \mathscr{W} \longrightarrow \mathbb{R}} \\
& w \longmapsto[w]=\frac{1}{2 \pi} \int_{0}^{2 \pi} e^{-i s} w(s, \epsilon, \nu) d s
\end{aligned}
$$

and the formal power series in the variable $\epsilon$ of the function $(s, \epsilon, \nu) \mapsto w(s, \epsilon, \nu)$, because

$$
\begin{aligned}
\epsilon & =\frac{1}{2 \pi} \int_{0}^{2 \pi} e^{-i s}\left(\sum_{k=1}^{\infty} \frac{1}{k !} w_{k}(s, \nu) \epsilon^{k}\right) d s \\
& =\sum_{k=1}^{\infty} \frac{1}{k !}\left(\frac{1}{2 \pi} \int_{0}^{2 \pi} e^{-i s} w_{k}(s, \nu) d s\right) \epsilon^{k}=\sum_{k=1}^{\infty} \frac{1}{k !}\left[w_{k}\right] \epsilon^{k} .
\end{aligned}
$$

The terms of the sequences $\left\{w_{k}(s, \nu)\right\}_{k \in \mathbb{N}},\left\{\mu_{k}(\nu)\right\}_{k \in \mathbb{N}}$ and $\left\{\omega_{k}(\nu)\right\}_{k \in \mathbb{N}}$ are determined through a process that involves analysis of the powers in $\epsilon$, obtained by replacing (29) into the differential equation (28). Note that, for $k=2,3, \ldots$ and $j=0, \ldots, k$, the coefficients of powers in $\epsilon$ are determined by expanding the composition $(\epsilon, \nu) \mapsto g_{k-j, j}(\phi(\epsilon, \nu), \nu)$ in the Taylor series around $\epsilon=0$. Such an expansion, up to the fifth-order terms, is of the following form:

$$
\begin{aligned}
& g_{k-j, j}(\phi(\epsilon, \nu), \nu) \\
& =g_{k-j, j}\left(\xi_{0}\right)+\mu_{1}(\nu) \partial_{\mu} g_{k-j, j}\left(\xi_{0}\right) \epsilon \\
& +\frac{1}{2}\left(\mu_{2}(\nu) \partial_{\mu} g_{k-j, j}\left(\xi_{0}\right)\right. \\
& +\frac{1}{6}\left(\mu_{3}(\nu) \partial_{\mu} g_{k-j, j}\left(\xi_{0}\right)+\mu_{1}(\nu)^{2} \partial_{\mu}^{2} g_{k-j, j}\left(\xi_{0}\right)\right) \epsilon^{2} \\
& +3 \mu_{1}(\nu) \mu_{2}(\nu) \partial_{\mu}^{2} g_{k-j, j}\left(\xi_{0}\right) \\
& \left.+\mu_{1}(\nu)^{3} \partial_{\mu}^{3} g_{k-j, j}\left(\xi_{0}\right)\right) \epsilon^{3} \\
& +\frac{1}{24}\left(\mu_{4}(\nu) \partial_{\mu} g_{k-j, j}\left(\xi_{0}\right)+3 \mu_{2}(\nu)^{2} \partial_{\mu}^{2} g_{k-j, j}\left(\xi_{0}\right)\right. \\
& +4 \mu_{1}(\nu) \mu_{2}(\nu) \partial_{\mu} g_{k-j, j}\left(\xi_{0}\right)+6 \mu_{1}(\nu)^{2} \mu_{2}(\nu) \\
& \left.\times \partial_{\mu}^{3} g_{k-j, j}\left(\xi_{0}\right)+\mu_{1}(\nu)^{4} \partial_{\mu}^{4} g_{k-j, j}\left(\xi_{0}\right)\right) \epsilon^{4} \\
& +\frac{1}{120}\left(\mu_{5}(\nu) \partial_{\mu} g_{k-j, j}\left(\xi_{0}\right)\right. \\
& +\left(10 \mu_{2}(\nu) \mu_{3}(\nu)+5 \mu_{1}(\nu) \mu_{4}(\nu)\right) \partial_{\mu}^{2} g_{k-j, j}\left(\xi_{0}\right) \\
& +\left(15 \mu_{1}(\nu) \mu_{2}(\nu)^{2}+10 \mu_{1}(\nu)^{2} \mu_{3}(\nu)\right) \\
& \times \partial_{\mu}^{3} g_{k-j, j}\left(\xi_{0}\right)+10 \mu_{1}(\nu)^{3} \mu_{2}(\nu) \partial_{\mu}^{4} g_{k-j, j}\left(\xi_{0}\right) \\
& \left.+\mu_{1}(\nu)^{5} \partial_{\mu}^{5} g_{k-j, j}\left(\xi_{0}\right)\right) \epsilon^{5} \\
& +O_{g}\left(\epsilon^{6}, v\right)
\end{aligned}
$$

with the same being valid for the composition $(\epsilon, \nu) \mapsto$ $\lambda(\phi(\epsilon, \nu), \nu)=\gamma(\phi(\epsilon, \nu), \nu)+i \eta(\phi(\epsilon, \nu), \nu)$.

The coefficient of the term in $\epsilon$ leads to the following boundary value problem:

$$
\begin{aligned}
& w_{1}^{\prime}(s, v)-i w_{1}(s, v)=0, \\
& w_{1}(s, v)=w_{1}(s+2 \pi, v) .
\end{aligned}
$$

The solution of the differential equation in (34) is

$$
w_{1}(s, v)=C_{1} e^{i s},
$$

and as by Proposition $1,\left[w_{1}\right]=1$, it follows that

$$
1=\left[w_{1}\right]=\left[C_{1} e^{i s}\right]=C_{1}\left[e^{i s}\right]=C_{1} .
$$

Thus,

$$
w_{1}(s, \nu)=e^{i s},
$$

which is a periodic function of period $2 \pi$ in the variable $s$. In fact, the terms of the sequence $\left\{w_{k}(s, \nu)\right\}_{k \in \mathbb{N}}$ are solutions of certain boundary value problems which appear when (29) is substituted into the differential equation (28). For each $k=$ $1,2, \ldots$, the boundary value problem is of the following form:

$$
\begin{aligned}
& w_{k+1}^{\prime}(s, \nu)-i w_{k+1}(s, \nu)=H_{k+1}\left(s, \mu_{k}(\nu), \omega_{k}(\nu)\right), \\
& w_{k+1}(s, \nu)=w_{k+1}(s+2 \pi, \nu),
\end{aligned}
$$

where $H_{k+1}\left(s, \mu_{k}(\nu), \omega_{k}(\nu)\right)=H_{k+1}\left(s+2 \pi, \mu_{k}(\nu), \omega_{k}(\nu)\right)$.

The following theorem guarantees the existence of the solutions of the boundary value problem (38).

Theorem 2. For each $k=1,2, \ldots$, the boundary value problem (38) admits solution if and only if

$$
\left[H_{k+1}\right]=0 \text {. }
$$

Proof. For fixed $k=1,2, \ldots$, suppose that $(s, \nu) \mapsto \varphi_{k+1}(s, \nu)$ is the solution of (38). Thus,

$$
\begin{aligned}
& \frac{1}{2 \pi} \int_{0}^{2 \pi} e^{-i s}\left(\varphi_{k+1}^{\prime}(s, \nu)-i \varphi_{k+1}(s, \nu)\right) d s \\
& \quad=\frac{1}{2 \pi} \int_{0}^{2 \pi} e^{-i s} H_{k+1}\left(s, \mu_{k}(\nu), \omega_{k}(\nu)\right) d s
\end{aligned}
$$

and by integrating by parts the left member of (40), it follows that $\left[H_{k+1}\right]=0$. Now suppose that $\left[H_{k+1}\right]=0$ for a fixed $k=1,2, \ldots$. The general solution $(s, v) \mapsto \varphi_{k+1}(s, v)$ of the differential equation in (38) is of the following form:

$$
\varphi_{k+1}(s, \nu)=e^{i s} \varphi_{0}^{k+1}+e^{i s} \int_{0}^{s} e^{-i \zeta} H_{k+1}\left(\zeta, \mu_{k}(\nu), \omega_{k}(\nu)\right) d \zeta
$$

where $\varphi_{0}^{k+1}=\varphi_{k+1}(0, \nu)$. This solution will be periodic of period $2 \pi$ if $\varphi_{k+1}(0, v)=\varphi_{0}^{k+1}=\varphi_{k+1}(2 \pi, \nu)$; that is, if

$$
\begin{aligned}
\varphi_{k+1}(0, \nu) & =\varphi_{k+1}(2 \pi, \nu) \\
& =\varphi_{0}^{k+1}+\int_{0}^{2 \pi} e^{-i \zeta} H_{k+1}\left(\zeta, \mu_{k}(\nu), \omega_{k}(\nu)\right) d \zeta \\
& =\varphi_{0}^{k+1}+2 \pi\left[H_{k+1}\right] .
\end{aligned}
$$


Thus, using the hypothesis $\left[H_{k+1}\right]=0$, it follows that $\varphi_{k+1}(s, \nu)=\varphi_{k+1}(s+2 \pi, \nu)$, and, therefore, for each fixed $k=1,2, \ldots$, the function $(s, v) \mapsto \varphi_{k+1}(s, v)$ is the solution of the boundary value problem (38).

The previous theorem shows that, for $k=1,2, \ldots$, the solution of (38) is obtained by solving the differential equation in (38) with conditions $\left[H_{k+1}\right]=0$ and $\left[w_{k+1}\right]=0$.

Continuing the process and using the result (37), the coefficient of the term in $\epsilon^{2}$ provides the boundary value problem

$$
\begin{aligned}
& w_{2}^{\prime}(s, v)-i w_{2}(s, \nu)=H_{2}\left(s, \mu_{1}(\nu), \omega_{1}(\nu)\right), \\
& w_{2}(s, v)=w_{2}(s+2 \pi, \nu),
\end{aligned}
$$

where

$$
\begin{gathered}
H_{2}\left(s, \mu_{1}(\nu), \omega_{1}(\nu)\right) \\
=\frac{1}{\omega_{0}(\nu)}\left(e^{2 i s} g_{2,0}\left(\xi_{0}\right)+2 g_{1,1}\left(\xi_{0}\right)+e^{-2 i s} g_{0,2}\left(\xi_{0}\right)\right. \\
\left.+2 e^{i s}\left(\mu_{1}(\nu) \partial_{\mu} \lambda\left(\xi_{0}\right)-i \omega_{1}(\nu)\right)\right) .
\end{gathered}
$$

By applying Theorem 2 to the function $\left(s, \mu_{1}(\nu), \omega_{1}(\nu)\right) \mapsto$ $H_{2}\left(s, \mu_{1}(\nu), \omega_{1}(\nu)\right)$, it follows that

$$
\left[H_{2}\right]=\mu_{1}(\nu)\left(\partial_{\mu} \gamma\left(\xi_{0}\right)+i \partial_{\mu} \eta\left(\xi_{0}\right)\right)-i \omega_{1}(\nu)=0
$$

and by separating the real and imaginary parts of (45), we have $\mu_{1}(\nu)=0$ and $\omega_{1}(\nu)=0$. Under these conditions, Theorem 2 guarantees the existence of the solution of the boundary value problem (43), which is given by

$$
\begin{aligned}
& w_{2}(s, \nu) \\
& =\frac{1}{3 i \omega_{0}(\nu)}\left(3 e^{2 i s} g_{2,0}\left(\xi_{0}\right)-6 g_{1,1}\left(\xi_{0}\right)-e^{-2 i s} g_{0,2}\left(\xi_{0}\right)\right) .
\end{aligned}
$$

For the coefficient of the term in $\epsilon^{3}$, we have the following boundary value problem:

$$
\begin{aligned}
& w_{3}^{\prime}(s, \nu)-i w_{3}(s, \nu)=H_{3}\left(s, \mu_{2}(\nu), \omega_{2}(\nu)\right), \\
& w_{3}(s, \nu)=w_{3}(s+2 \pi, \nu),
\end{aligned}
$$

with

$$
\begin{aligned}
& H_{3}\left(s, \mu_{2}(\nu), \omega_{2}(\nu)\right) \\
& =\frac{3}{\omega_{0}(\nu)}\left(H_{3}^{3}\left(\xi_{0}\right) e^{3 i s}+H_{3}^{1}\left(\xi_{0}\right) e^{i s}\right. \\
& \left.\quad+H_{3}^{-1}\left(\xi_{0}\right) e^{-i s}+H_{3}^{-3}\left(\xi_{0}\right) e^{-3 i s}\right),
\end{aligned}
$$

where

$$
\begin{gathered}
H_{3}^{3}\left(\xi_{0}\right)=\frac{1}{3} g_{3,0}\left(\xi_{0}\right)-\frac{i g_{2,0}\left(\xi_{0}\right)^{2}}{\omega_{0}(\nu)}-\frac{i g_{1,1}\left(\xi_{0}\right) \bar{g}_{0,2}\left(\xi_{0}\right)}{\omega_{0}(\nu)} \\
H_{3}^{1}\left(\xi_{0}\right)=\mu_{2}(\nu) \partial_{\mu} \lambda\left(\xi_{0}\right)-i \omega_{2}(\nu)+G_{2,1}\left(\xi_{0}\right), \\
H_{3}^{-1}\left(\xi_{0}\right)=\frac{2 i g_{1,1}\left(\xi_{0}\right)^{2}}{\omega_{0}(\nu)}+\frac{i \bar{g}_{2,0}\left(\xi_{0}\right) g_{1,1}\left(\xi_{0}\right)}{\omega_{0}(\nu)}+g_{1,2}\left(\xi_{0}\right) \\
+\frac{i g_{2,0}\left(\xi_{0}\right) g_{0,2}\left(\xi_{0}\right)}{3 \omega_{0}(\nu)}-\frac{2 i g_{0,2}\left(\xi_{0}\right) \bar{g}_{1,1}\left(\xi_{0}\right)}{\omega_{0}(\nu)} \\
H_{3}^{-3}\left(\xi_{0}\right)=\frac{1}{3} g_{0,3}\left(\xi_{0}\right)+\frac{i g_{1,1}\left(\xi_{0}\right) g_{0,2}\left(\xi_{0}\right)}{3 \omega_{0}(\nu)} \\
+\frac{i g_{0,2}\left(\xi_{0}\right) \bar{g}_{2,0}\left(\xi_{0}\right)}{\omega_{0}(\nu)}
\end{gathered}
$$

and the coefficient $G_{2,1}\left(\xi_{0}\right)$ is defined as

$$
\begin{aligned}
G_{2,1}\left(\xi_{0}\right)= & \frac{i g_{2,0}\left(\xi_{0}\right) g_{1,1}\left(\xi_{0}\right)+\omega_{0}(\nu) g_{2,1}\left(\xi_{0}\right)}{\omega_{0}(\nu)} \\
& -\frac{2 i\left|g_{1,1}\left(\xi_{0}\right)\right|^{2}}{\omega_{0}(\nu)}-\frac{i\left|g_{0,2}\left(\xi_{0}\right)\right|^{2}}{3 \omega_{0}(\nu)} .
\end{aligned}
$$

Expression (50) is identical to the one given in [1].

Continuing the process and calculating $\left[\mathrm{H}_{3}\right]$, it follows that

$$
\left[H_{3}\right]=\mu_{2}(\nu)\left(\partial_{\mu} \gamma\left(\xi_{0}\right)+i \partial_{\mu} \eta\left(\xi_{0}\right)\right)-i \omega_{2}(\nu)+G_{2,1}\left(\xi_{0}\right)=0
$$

And by separating the real and imaginary parts,

$$
\begin{gathered}
\mu_{2}(\nu)=-\frac{\operatorname{Re}\left(G_{2,1}\left(\xi_{0}\right)\right)}{\partial_{\mu} \gamma\left(\xi_{0}\right)}, \\
\omega_{2}(\nu)=\operatorname{Im}\left(G_{2,1}\left(\xi_{0}\right)\right)+\mu_{2}(\nu) \partial_{\mu} \eta\left(\xi_{0}\right) .
\end{gathered}
$$

Once the coefficients $\mu_{2}(\nu)$ and $\omega_{2}(\nu)$ are determined, the solution of the boundary value problem (47) has the following form:

$$
\begin{aligned}
& w_{3}(s, \nu) \\
& =\frac{1}{4 \omega_{0}(\nu)^{2}}\left(w_{3}^{3}\left(\xi_{0}\right) e^{3 i s}+w_{3}^{-1}\left(\xi_{0}\right) e^{-i s}+w_{3}^{-3}\left(\xi_{0}\right) e^{-3 i s}\right),
\end{aligned}
$$


where

$$
\begin{aligned}
& w_{3}^{3}\left(\xi_{0}\right)=-6 g_{2,0}\left(\xi_{0}\right)^{2}-2 i \omega_{0}(\nu) g_{3,0}\left(\xi_{0}\right) \\
&- 2 g_{1,1}\left(\xi_{0}\right) \bar{g}_{0,2}\left(\xi_{0}\right) \\
& w_{3}^{-1}\left(\xi_{0}\right)=-12 g_{1,1}\left(\xi_{0}\right)^{2}-6 \bar{g}_{2,0}\left(\xi_{0}\right) g_{1,1}\left(\xi_{0}\right) \\
&+6 i \omega_{0}(\nu) g_{1,2}\left(\xi_{0}\right)-2 g_{0,2}\left(\xi_{0}\right) g_{2,0}\left(\xi_{0}\right) \\
&+12 g_{0,2}\left(\xi_{0}\right) \bar{g}_{1,1}\left(\xi_{0}\right) \\
& w_{3}^{-3}\left(\xi_{0}\right)=i \omega_{0}(\nu) g_{0,3}\left(\xi_{0}\right)-g_{0,2}\left(\xi_{0}\right) g_{1,1}\left(\xi_{0}\right) \\
&- 3 g_{0,2}\left(\xi_{0}\right) \bar{g}_{2,0}\left(\xi_{0}\right) .
\end{aligned}
$$

Definition 3. The real number

$$
\begin{aligned}
l_{1}\left(\xi_{0}\right) & =\frac{1}{2} \operatorname{Re}\left(G_{2,1}\left(\xi_{0}\right)\right) \\
& =\frac{1}{2 \omega_{0}(\nu)} \operatorname{Re}\left(i g_{2,0}\left(\xi_{0}\right) g_{1,1}\left(\xi_{0}\right)+\omega_{0}(\nu) g_{2,1}\left(\xi_{0}\right)\right)
\end{aligned}
$$

is called the first Lyapunov coefficient.

Remark 4. A Hopf point of codimension one for (1) is an equilibrium point $\left(\mathbf{0}, \xi_{0}\right) \in W \times U$, with $\xi_{0}=(0, v)$, such that $A\left(\xi_{0}\right)=D f\left(\mathbf{0}, \xi_{0}\right)$ has eigenvalues $\lambda$ and $\bar{\lambda}$, with $\lambda\left(\xi_{0}\right)=$ $\gamma\left(\xi_{0}\right)+i \eta\left(\xi_{0}\right), \gamma\left(\xi_{0}\right)=0, \eta\left(\xi_{0}\right)=\omega_{0}(\nu)>0$, and the first Lyapunov coefficient, $l_{1}\left(\xi_{0}\right) \in \mathbb{R}$, is different from zero. A transversal Hopf point of codimension one is a Hopf point of codimension one such that

$$
\partial_{\mu} \gamma\left(\xi_{0}\right) \neq 0
$$

for $\xi_{0} \in U$. In a neighborhood of a transversal Hopf point of codimension one $\left(\mathbf{0}, \xi_{0}\right) \in W \times U$, with $l_{1}\left(\xi_{0}\right) \neq 0$, the dynamic behavior of differential equation (1) is orbitally topologically equivalent to the following complex normal form:

$$
w^{\prime}=(\alpha+i) w+s w|w|^{2}
$$

where $s=\operatorname{sign}\left(l_{1}\left(\xi_{0}\right)\right)$. The sign of the first Lyapunov coefficient determines the stability of the family of periodic orbits that appears (or disappears) from $\left(\mathbf{0}, \xi_{0}\right) \in W \times U$ as will be seen later.

When $l_{1}\left(\xi_{1}\right)=0$, for $\xi_{1}=(0,0) \in U$, there is the possibility of Hopf bifurcations of codimension two. In this case, it is necessary to obtain an expression for $G_{3,2}\left(\xi_{1}\right)$.

Applying Theorem 2 to the boundary value problem for $k=3$, it follows that $\mu_{3}(\nu)=0, \omega_{3}(\nu)=0$ and

$$
\begin{array}{r}
w_{4}(s, \nu)=\frac{1}{45 \omega_{0}(\nu)^{3}}\left(w_{4}^{4}\left(\xi_{0}\right) e^{4 i s}+w_{4}^{2}\left(\xi_{0}\right) e^{2 i s}+w_{4}^{0}\left(\xi_{0}\right)\right. \\
\left.+w_{4}^{-2}\left(\xi_{0}\right) e^{-2 i s}+w_{4}^{-4}\left(\xi_{0}\right) e^{-4 i s}\right)
\end{array}
$$

where

$$
\begin{aligned}
& w_{4}^{4}\left(\xi_{0}\right)=135 i g_{2,0}\left(\xi_{0}\right)^{3}-120 \omega_{0}(\nu) g_{3,0}\left(\xi_{0}\right) g_{2,0}\left(\xi_{0}\right) \\
& +105 i g_{1,1}\left(\xi_{0}\right) \bar{g}_{0,2}\left(\xi_{0}\right) g_{2,0}\left(\xi_{0}\right) \\
& +5 i g_{0,2}\left(\xi_{0}\right) \bar{g}_{0,2}\left(\xi_{0}\right)^{2}-15 i \omega_{0}(\nu)^{2} g_{4,0}\left(\xi_{0}\right) \\
& -30 \omega_{0}(\nu) g_{2,1}\left(\xi_{0}\right) \bar{g}_{0,2}\left(\xi_{0}\right) \\
& -15 \omega_{0}(\nu) g_{1,1}\left(\xi_{0}\right) \bar{g}_{0,3}\left(\xi_{0}\right) \\
& +15 i g_{1,1}\left(\xi_{0}\right) \bar{g}_{0,2}\left(\xi_{0}\right) \bar{g}_{1,1}\left(\xi_{0}\right) \\
& w_{4}^{2}\left(\xi_{0}\right)=-180 i g_{3,1}\left(\xi_{0}\right) \omega_{0}(\nu)^{2} \\
& -270 i \mu_{2}(\nu) \partial_{\mu} g_{2,0}\left(\xi_{0}\right) \omega_{0}(\nu)^{2} \\
& +450 g_{1,1}\left(\xi_{0}\right) g_{3,0}\left(\xi_{0}\right) \omega_{0}(\nu) \\
& -180 g_{1,2}\left(\xi_{0}\right) \bar{g}_{0,2}\left(\xi_{0}\right) \omega_{0}(\nu) \\
& -45 g_{0,2}\left(\xi_{0}\right) \bar{g}_{0,3}\left(\xi_{0}\right) \omega_{0}(\nu) \\
& -540 g_{2,1}\left(\xi_{0}\right) \bar{g}_{1,1}\left(\xi_{0}\right) \omega_{0}(\nu) \\
& -270 g_{1,1}\left(\xi_{0}\right) \bar{g}_{1,2}\left(\xi_{0}\right) \omega_{0}(\nu) \\
& +270 \mu_{2}(\nu) g_{2,0}\left(\xi_{0}\right) \partial_{\mu} \gamma\left(\xi_{0}\right) \omega_{0}(\nu) \\
& +270 i \mu_{2}(\nu) g_{2,0}\left(\xi_{0}\right) \partial_{\mu} \eta\left(\xi_{0}\right) \omega_{0}(\nu) \\
& +270 i g_{1,1}\left(\xi_{0}\right) g_{2,0}\left(\xi_{0}\right)^{2} \\
& +540 i g_{1,1}\left(\xi_{0}\right) \bar{g}_{1,1}\left(\xi_{0}\right)^{2} \\
& -630 i g_{1,1}\left(\xi_{0}\right)^{2} \bar{g}_{0,2}\left(\xi_{0}\right) \\
& -45 i g_{0,2}\left(\xi_{0}\right) g_{2,0}\left(\xi_{0}\right) \bar{g}_{0,2}\left(\xi_{0}\right) \\
& -270 i g_{1,1}\left(\xi_{0}\right) g_{2,0}\left(\xi_{0}\right) \bar{g}_{1,1}\left(\xi_{0}\right) \\
& +225 i g_{0,2}\left(\xi_{0}\right) \bar{g}_{0,2}\left(\xi_{0}\right) \bar{g}_{1,1}\left(\xi_{0}\right) \\
& +90 i g_{1,1}\left(\xi_{0}\right) \bar{g}_{0,2}\left(\xi_{0}\right) \bar{g}_{2,0}\left(\xi_{0}\right) \text {, } \\
& w_{4}^{0}\left(\xi_{0}\right)=270 i g_{2,2}\left(\xi_{0}\right) \omega_{0}(\nu)^{2} \\
& +540 i \mu_{2}(\nu) \partial_{\mu} g_{1,1}\left(\xi_{0}\right) \omega_{0}(\nu)^{2} \\
& -1080 g_{1,1}\left(\xi_{0}\right) g_{2,1}\left(\xi_{0}\right) \omega_{0}(\nu) \\
& -90 g_{0,2}\left(\xi_{0}\right) g_{3,0}\left(\xi_{0}\right) \omega_{0}(\nu) \\
& +90 g_{0,3}\left(\xi_{0}\right) \bar{g}_{0,2}\left(\xi_{0}\right) \omega_{0}(\nu) \\
& +1080 g_{1,2}\left(\xi_{0}\right) \bar{g}_{1,1}\left(\xi_{0}\right) \omega_{0}(\nu) \\
& +270 g_{0,2}\left(\xi_{0}\right) \bar{g}_{1,2}\left(\xi_{0}\right) \omega_{0}(\nu) \\
& -270 g_{2,1}\left(\xi_{0}\right) \bar{g}_{2,0}\left(\xi_{0}\right) \omega_{0}(\nu) \\
& -540 \mu_{2}(\nu) g_{1,1}\left(\xi_{0}\right) \partial_{\mu} \gamma\left(\xi_{0}\right) \omega_{0}(\nu)
\end{aligned}
$$




$$
\begin{aligned}
& -540 i \mu_{2}(\nu) g_{1,1}\left(\xi_{0}\right) \partial_{\mu} \eta\left(\xi_{0}\right) \omega_{0}(\nu) \\
& -1080 i g_{0,2}\left(\xi_{0}\right) \bar{g}_{1,1}\left(\xi_{0}\right)^{2} \\
& -1080 i g_{1,1}\left(\xi_{0}\right)^{2} g_{2,0}\left(\xi_{0}\right) \\
& +570 i g_{0,2}\left(\xi_{0}\right) g_{1,1}\left(\xi_{0}\right) \bar{g}_{0,2}\left(\xi_{0}\right) \\
& +1080 i g_{1,1}\left(\xi_{0}\right)^{2} \bar{g}_{1,1}\left(\xi_{0}\right) \\
& +270 i g_{0,2}\left(\xi_{0}\right) g_{2,0}\left(\xi_{0}\right) \bar{g}_{1,1}\left(\xi_{0}\right) \text {, } \\
& w_{4}^{-2}\left(\xi_{0}\right)=-180 i g_{1,1}\left(\xi_{0}\right)^{3}-270 i \bar{g}_{2,0}\left(\xi_{0}\right) g_{1,1}\left(\xi_{0}\right)^{2} \\
& -90 i \bar{g}_{2,0}\left(\xi_{0}\right)^{2} g_{1,1}\left(\xi_{0}\right) \\
& -270 \omega_{0}(\nu) g_{1,2}\left(\xi_{0}\right) g_{1,1}\left(\xi_{0}\right) \\
& -165 i g_{0,2}\left(\xi_{0}\right) g_{2,0}\left(\xi_{0}\right) g_{1,1}\left(\xi_{0}\right) \\
& +330 i g_{0,2}\left(\xi_{0}\right) \bar{g}_{1,1}\left(\xi_{0}\right) g_{1,1}\left(\xi_{0}\right) \\
& -30 \omega_{0}(\nu) \bar{g}_{3,0}\left(\xi_{0}\right) g_{1,1}\left(\xi_{0}\right) \\
& +60 i \omega_{0}(\nu)^{2} g_{1,3}\left(\xi_{0}\right) \\
& -15 \omega_{0}(\nu) g_{0,3}\left(\xi_{0}\right) g_{2,0}\left(\xi_{0}\right) \\
& -120 \omega_{0}(\nu) g_{0,2}\left(\xi_{0}\right) g_{2,1}\left(\xi_{0}\right) \\
& +20 i g_{0,2}\left(\xi_{0}\right)^{2} \bar{g}_{0,2}\left(\xi_{0}\right) \\
& +180 \omega_{0}(\nu) g_{0,3}\left(\xi_{0}\right) \bar{g}_{1,1}\left(\xi_{0}\right) \\
& -180 \omega_{0}(\nu) g_{1,2}\left(\xi_{0}\right) \bar{g}_{2,0}\left(\xi_{0}\right) \\
& -45 i g_{0,2}\left(\xi_{0}\right) g_{2,0}\left(\xi_{0}\right) \bar{g}_{2,0}\left(\xi_{0}\right) \\
& +180 i g_{0,2}\left(\xi_{0}\right) \bar{g}_{1,1}\left(\xi_{0}\right) \bar{g}_{2,0}\left(\xi_{0}\right) \\
& -90 \mu_{2}(\nu) \omega_{0}(\nu) g_{0,2}\left(\xi_{0}\right) \partial_{\mu} \gamma\left(\xi_{0}\right) \\
& -90 i \mu_{2}(\nu) \omega_{0}(\nu) g_{0,2}\left(\xi_{0}\right) \partial_{\mu} \eta\left(\xi_{0}\right) \\
& +90 i \mu_{2}(\nu) \omega_{0}(\nu)^{2} \partial_{\mu} g_{0,2}\left(\xi_{0}\right) \\
& w_{4}^{-4}\left(\xi_{0}\right)=9 i g_{0,4}\left(\xi_{0}\right) \omega_{0}(\nu)^{2}-9 g_{0,3}\left(\xi_{0}\right) g_{1,1}\left(\xi_{0}\right) \omega_{0}(\nu) \\
& -18 g_{0,2}\left(\xi_{0}\right) g_{1,2}\left(\xi_{0}\right) \omega_{0}(\nu) \\
& -9 i g_{0,2}\left(\xi_{0}\right) g_{1,1}\left(\xi_{0}\right)^{2} \\
& -54 g_{0,3}\left(\xi_{0}\right) \bar{g}_{2,0}\left(\xi_{0}\right) \omega_{0}(\nu) \\
& -18 g_{0,2}\left(\xi_{0}\right) \bar{g}_{3,0}\left(\xi_{0}\right) \omega_{0} \\
& -81 i g_{0,2}\left(\xi_{0}\right) \bar{g}_{2,0}\left(\xi_{0}\right)^{2}
\end{aligned}
$$

$$
\begin{aligned}
& -3 i g_{0,2}\left(\xi_{0}\right)^{2} g_{2,0}\left(\xi_{0}\right) \\
& -18 i g_{0,2}\left(\xi_{0}\right)^{2} \bar{g}_{1,1}\left(\xi_{0}\right) \\
& -45 i g_{0,2}\left(\xi_{0}\right) g_{1,1}\left(\xi_{0}\right) \bar{g}_{2,0}\left(\xi_{0}\right) .
\end{aligned}
$$

From the boundary value problem for $k=5$, it follows that

$$
\begin{aligned}
\mu_{4}(\nu)= & -\left(2 \operatorname{Re}\left(G_{3,2}\left(\xi_{0}\right)\right)+6 \mu_{2}(\nu) \operatorname{Re}\left(\partial_{\mu} G_{2,1}\left(\xi_{0}\right)\right)\right. \\
& \left.+3 \mu_{2}(\nu)^{2} \partial_{\mu}^{2} \gamma\left(\xi_{0}\right)\right) \\
& \times\left(\partial_{\mu} \gamma\left(\xi_{0}\right)\right)^{-1}, \\
\omega_{4}(\nu)= & 2 \operatorname{Im}\left(G_{3,2}\left(\xi_{0}\right)\right)+\mu_{4}(\nu) \partial_{\mu} \eta\left(\xi_{0}\right) \\
& +6 \mu_{2}(\nu) \operatorname{Im}\left(\partial_{\mu} G_{2,1}\left(\xi_{0}\right)\right)+3 \mu_{2}(\nu)^{2} \partial_{\mu}^{2} \eta\left(\xi_{0}\right),
\end{aligned}
$$

where

$$
\begin{aligned}
& \partial_{\mu} G_{2,1}\left(\xi_{0}\right)=\frac{3 g_{1,1}\left(\xi_{0}\right) g_{2,0}\left(\xi_{0}\right) \partial_{\mu} \gamma\left(\xi_{0}\right)}{\omega_{0}(\nu)^{2}} \\
& +\frac{g_{0,2}\left(\xi_{0}\right) \bar{g}_{0,2}\left(\xi_{0}\right) \partial_{\mu} \gamma\left(\xi_{0}\right)}{9 \omega_{0}(\nu)^{2}} \\
& +\frac{2 g_{1,1}\left(\xi_{0}\right) \bar{g}_{1,1}\left(\xi_{0}\right) \partial_{\mu} \gamma\left(\xi_{0}\right)}{\omega_{0}(\nu)^{2}} \\
& -\frac{i g_{1,1}\left(\xi_{0}\right) g_{2,0}\left(\xi_{0}\right) \partial_{\mu} \eta\left(\xi_{0}\right)}{\omega_{0}(\nu)^{2}} \\
& +\frac{i g_{0,2}\left(\xi_{0}\right) \bar{g}_{0,2}\left(\xi_{0}\right) \partial_{\mu} \eta\left(\xi_{0}\right)}{3 \omega_{0}(\nu)^{2}} \\
& +\frac{2 i g_{1,1}\left(\xi_{0}\right) \bar{g}_{1,1}\left(\xi_{0}\right) \partial_{\mu} \eta\left(\xi_{0}\right)}{\omega_{0}(\nu)^{2}} \\
& -\frac{i \bar{g}_{0,2}\left(\xi_{0}\right) \partial_{\mu} g_{0,2}\left(\xi_{0}\right)}{3 \omega_{0}(\nu)} \\
& +\frac{i g_{2,0}\left(\xi_{0}\right) \partial_{\mu} g_{1,1}\left(\xi_{0}\right)}{\omega_{0}(\nu)} \\
& -\frac{2 i \bar{g}_{1,1}\left(\xi_{0}\right) \partial_{\mu} g_{1,1}\left(\xi_{0}\right)}{\omega_{0}(\nu)} \\
& +\frac{i g_{1,1}\left(\xi_{0}\right) \partial_{\mu} g_{2,0}\left(\xi_{0}\right)}{\omega_{0}(\nu)}+\partial_{\mu} g_{2,1}\left(\xi_{0}\right) \\
& -\frac{i g_{0,2}\left(\xi_{0}\right) \partial_{\mu} \bar{g}_{0,2}\left(\xi_{0}\right)}{3 \omega_{0}(\nu)} \\
& -\frac{2 i g_{1,1}\left(\xi_{0}\right) \partial_{\mu} \bar{g}_{1,1}\left(\xi_{0}\right)}{\omega_{0}(\nu)}
\end{aligned}
$$




$$
\begin{aligned}
& G_{3,2}\left(\xi_{0}\right)=\frac{12 i \bar{g}_{0,2}\left(\xi_{0}\right) g_{1,1}\left(\xi_{0}\right)^{3}}{\omega_{0}(\nu)^{3}} \\
& -\frac{12 i \bar{g}_{1,1}\left(\xi_{0}\right)^{2} g_{1,1}\left(\xi_{0}\right)^{2}}{\omega_{0}(\nu)^{3}}-\frac{4 g_{3,0}\left(\xi_{0}\right) g_{1,1}\left(\xi_{0}\right)^{2}}{\omega_{0}(\nu)^{2}} \\
& +\frac{12 i g_{2,0}\left(\xi_{0}\right) \bar{g}_{1,1}\left(\xi_{0}\right) g_{1,1}\left(\xi_{0}\right)^{2}}{\omega_{0}(\nu)^{3}} \\
& +\frac{12 \bar{g}_{1,2}\left(\xi_{0}\right) g_{1,1}\left(\xi_{0}\right)^{2}}{\omega_{0}(\nu)^{2}} \\
& -\frac{3 i \bar{g}_{0,2}\left(\xi_{0}\right) \bar{g}_{2,0}\left(\xi_{0}\right) g_{1,1}\left(\xi_{0}\right)^{2}}{\omega_{0}(\nu)^{3}} \\
& +\frac{4 i g_{3,1}\left(\xi_{0}\right) g_{1,1}\left(\xi_{0}\right)}{\omega_{0}(\nu)} \\
& +\frac{9 g_{1,2}\left(\xi_{0}\right) \bar{g}_{0,2}\left(\xi_{0}\right) g_{1,1}\left(\xi_{0}\right)}{\omega_{0}(\nu)^{2}} \\
& +\frac{17 i g_{0,2}\left(\xi_{0}\right) g_{2,0}\left(\xi_{0}\right) \bar{g}_{0,2}\left(\xi_{0}\right) g_{1,1}\left(\xi_{0}\right)}{4 \omega_{0}(\nu)^{3}} \\
& +\frac{31 g_{0,2}\left(\xi_{0}\right) \bar{g}_{0,3}\left(\xi_{0}\right) g_{1,1}\left(\xi_{0}\right)}{12 \omega_{0}(\nu)^{2}} \\
& -\frac{175 i g_{0,2}\left(\xi_{0}\right) \bar{g}_{0,2}\left(\xi_{0}\right) \bar{g}_{1,1}\left(\xi_{0}\right) g_{1,1}\left(\xi_{0}\right)}{12 \omega_{0}(\nu)^{3}} \\
& +\frac{12 g_{2,1}\left(\xi_{0}\right) \bar{g}_{1,1}\left(\xi_{0}\right) g_{1,1}\left(\xi_{0}\right)}{\omega_{0}(\nu)^{2}} \\
& +\frac{6 i \bar{g}_{1,1}\left(\xi_{0}\right)^{2} \bar{g}_{2,0}\left(\xi_{0}\right) g_{1,1}\left(\xi_{0}\right)}{\omega_{0}(\nu)^{3}} \\
& -\frac{g_{3,0}\left(\xi_{0}\right) \bar{g}_{2,0}\left(\xi_{0}\right) g_{1,1}\left(\xi_{0}\right)}{\omega_{0}(v)^{2}} \\
& -\frac{6 i g_{2,0}\left(\xi_{0}\right) \bar{g}_{1,1}\left(\xi_{0}\right) \bar{g}_{2,0}\left(\xi_{0}\right) g_{1,1}\left(\xi_{0}\right)}{\omega_{0}(\nu)^{3}} \\
& +\frac{3 g_{2,0}\left(\xi_{0}\right) \bar{g}_{2,1}\left(\xi_{0}\right) g_{1,1}\left(\xi_{0}\right)}{\omega_{0}(\nu)^{2}} \\
& -\frac{3 i \bar{g}_{2,2}\left(\xi_{0}\right) g_{1,1}\left(\xi_{0}\right)}{\omega_{0}(\nu)} \\
& -\frac{6 \bar{g}_{1,1}\left(\xi_{0}\right) \bar{g}_{2,1}\left(\xi_{0}\right) g_{1,1}\left(\xi_{0}\right)}{\omega_{0}(\nu)^{2}} \\
& -\frac{\bar{g}_{0,2}\left(\xi_{0}\right) \bar{g}_{3,0}\left(\xi_{0}\right) g_{1,1}\left(\xi_{0}\right)}{\omega_{0}(\nu)^{2}} \\
& +\frac{8 i g_{0,2}\left(\xi_{0}\right) \bar{g}_{1,1}\left(\xi_{0}\right)^{3}}{\omega_{0}(\nu)^{3}}
\end{aligned}
$$$$
-\frac{2 i g_{0,2}\left(\xi_{0}\right)^{2} \bar{g}_{0,2}\left(\xi_{0}\right)^{2}}{9 \omega_{0}(\nu)^{3}}
$$$$
\begin{aligned}
& -\frac{12 g_{1,2}\left(\xi_{0}\right) \bar{g}_{1,1}\left(\xi_{0}\right)^{2}}{\omega_{0}(\nu)^{2}} \\
& -\frac{6 i g_{0,2}\left(\xi_{0}\right) g_{2,0}\left(\xi_{0}\right) \bar{g}_{1,1}\left(\xi_{0}\right)^{2}}{\omega_{0}(\nu)^{3}}
\end{aligned}
$$$$
+\frac{i g_{1,2}\left(\xi_{0}\right) g_{3,0}\left(\xi_{0}\right)}{\omega_{0}(\nu)}
$$$$
-\frac{g_{0,2}\left(\xi_{0}\right) g_{2,0}\left(\xi_{0}\right) g_{3,0}\left(\xi_{0}\right)}{3 \omega_{0}(\nu)^{2}}
$$$$
+g_{3,2}\left(\xi_{0}\right)+\frac{i g_{0,2}\left(\xi_{0}\right) g_{4,0}\left(\xi_{0}\right)}{3 \omega_{0}(\nu)}
$$$$
-\frac{i g_{1,3}\left(\xi_{0}\right) \bar{g}_{0,2}\left(\xi_{0}\right)}{\omega_{0}(\nu)}
$$$$
+\frac{g_{0,3}\left(\xi_{0}\right) g_{2,0}\left(\xi_{0}\right) \bar{g}_{0,2}\left(\xi_{0}\right)}{4 \omega_{0}(\nu)^{2}}
$$$$
+\frac{g_{0,2}\left(\xi_{0}\right) g_{2,1}\left(\xi_{0}\right) \bar{g}_{0,2}\left(\xi_{0}\right)}{\omega_{0}(v)^{2}}
$$$$
-\frac{i g_{0,3}\left(\xi_{0}\right) \bar{g}_{0,3}\left(\xi_{0}\right)}{4 \omega_{0}(v)}
$$$$
+\frac{i g_{0,2}\left(\xi_{0}\right) g_{2,0}\left(\xi_{0}\right)^{2} \bar{g}_{1,1}\left(\xi_{0}\right)}{\omega_{0}(v)^{3}}
$$$$
+\frac{3 g_{1,2}\left(\xi_{0}\right) g_{2,0}\left(\xi_{0}\right) \bar{g}_{1,1}\left(\xi_{0}\right)}{\omega_{0}(\nu)^{2}}
$$$$
-\frac{6 i g_{2,2}\left(\xi_{0}\right) \bar{g}_{1,1}\left(\xi_{0}\right)}{\omega_{0}(\nu)}
$$$$
+\frac{8 g_{0,2}\left(\xi_{0}\right) g_{3,0}\left(\xi_{0}\right) \bar{g}_{1,1}\left(\xi_{0}\right)}{3 \omega_{0}(v)^{2}}
$$$$
-\frac{9 g_{0,3}\left(\xi_{0}\right) \bar{g}_{0,2}\left(\xi_{0}\right) \bar{g}_{1,1}\left(\xi_{0}\right)}{4 \omega_{0}(v)^{2}}
$$$$
-\frac{3 i g_{1,2}\left(\xi_{0}\right) \bar{g}_{1,2}\left(\xi_{0}\right)}{\omega_{0}(v)}
$$$$
+\frac{g_{0,2}\left(\xi_{0}\right) g_{2,0}\left(\xi_{0}\right) \bar{g}_{1,2}\left(\xi_{0}\right)}{\omega_{0}(\nu)^{2}}
$$$$
-\frac{6 g_{0,2}\left(\xi_{0}\right) \bar{g}_{1,1}\left(\xi_{0}\right) \bar{g}_{1,2}\left(\xi_{0}\right)}{\omega_{0}(\nu)^{2}}
$$$$
-\frac{2 i g_{0,2}\left(\xi_{0}\right) \bar{g}_{1,3}\left(\xi_{0}\right)}{3 \omega_{0}(\nu)}+\frac{i g_{3,1}\left(\xi_{0}\right) \bar{g}_{2,0}\left(\xi_{0}\right)}{\omega_{0}(v)}
$$ 


$$
\begin{aligned}
& -\frac{i g_{0,2}\left(\xi_{0}\right) g_{2,0}\left(\xi_{0}\right) \bar{g}_{0,2}\left(\xi_{0}\right) \bar{g}_{2,0}\left(\xi_{0}\right)}{4 \omega_{0}(\nu)^{3}} \\
& +\frac{g_{0,2}\left(\xi_{0}\right) \bar{g}_{0,3}\left(\xi_{0}\right) \bar{g}_{2,0}\left(\xi_{0}\right)}{12 \omega_{0}(\nu)^{2}} \\
& +\frac{23 i g_{0,2}\left(\xi_{0}\right) \bar{g}_{0,2}\left(\xi_{0}\right) \bar{g}_{1,1}\left(\xi_{0}\right) \bar{g}_{2,0}\left(\xi_{0}\right)}{12 \omega_{0}(\nu)^{3}} \\
& -\frac{2 g_{0,2}\left(\xi_{0}\right) \bar{g}_{0,2}\left(\xi_{0}\right) \bar{g}_{2,1}\left(\xi_{0}\right)}{3 \omega_{0}(\nu)^{2}} .
\end{aligned}
$$

Rewriting the coefficient $G_{3,2}\left(\xi_{0}\right)$ in a convenient way, expression (64) is exactly the one that appears in [1].

Definition 5. The real number

$$
l_{2}\left(\xi_{0}\right)=\frac{1}{12} \operatorname{Re}\left(G_{3,2}\left(\xi_{0}\right)\right),
$$

where $G_{3,2}\left(\xi_{0}\right)$ is given in (64), is called the second Lyapunov coefficient.

Remark 6. A Hopf point of codimension two for (1) is an equilibrium point $\left(\mathbf{0}, \xi_{1}\right) \in W \times U$, where $\xi_{1}=(0,0)$, that satisfies the definition of a point Hopf of codimension one, except that $l_{1}\left(\xi_{1}\right)=0$. Moreover, it satisfies an additional condition; the second Lyapunov coefficient $l_{2}\left(\xi_{1}\right)$ is nonzero. A Hopf point of codimension two is transversal if

$$
\partial_{\mu} \gamma\left(\xi_{1}\right) \operatorname{Re}\left(\partial_{\nu} G_{2,1}\left(\xi_{1}\right)\right) \neq 0 .
$$

In a neighborhood of a transversal Hopf point of codimension two $\left(\mathbf{0}, \xi_{1}\right) \in W \times U$, with $l_{2}\left(\xi_{1}\right) \neq 0$, the dynamic behavior of differential equation (1) is orbitally topologically equivalent to the following complex normal form:

$$
w^{\prime}=(\alpha+i) w+\beta w|w|^{2}+s w|w|^{4},
$$

where $s=\operatorname{sign}\left(l_{2}\left(\xi_{1}\right)\right)$. In the bifurcation diagram of (67), there exists a curve of nonhyperbolic periodic orbits that has the exact representations

$$
\Gamma(\epsilon)=\left(s \epsilon^{4},-2 s \epsilon^{2}\right),
$$

as a curve parameterized by $\epsilon$ or as a graph of the function

$$
\alpha=\Lambda(\beta)=\frac{1}{4 s} \beta^{2},
$$

for $\beta \geq 0$.

The function $(s, \nu) \mapsto w_{5}(s, \nu)$ will not be shown here because it is a long expression and it is not necessary in this work. In many results in this section and, particularly in (63), the following expressions $\partial_{\mu} \gamma\left(\xi_{0}\right), \partial_{\mu} \eta\left(\xi_{0}\right), \partial_{\mu}^{2} \gamma\left(\xi_{0}\right), \partial_{\mu}^{2} \eta\left(\xi_{0}\right)$, $\partial_{\mu} g_{2,0}\left(\xi_{0}\right), \partial_{\mu} g_{1,1}\left(\xi_{0}\right), \partial_{\mu} g_{0,2}\left(\xi_{0}\right)$, and $\partial_{\mu} g_{2,1}\left(\xi_{0}\right)$ appear. These expressions are calculated according to Propositions 7 and 8 .
Proposition 7. Consider the differential equation (1) with an equilibrium point $(\mathbf{0}, \xi) \in W \times U$, such that the linear part of the map $(\mathbf{x}, \xi) \mapsto f(\mathbf{x}, \xi)$, evaluated at $\left(\mathbf{0}, \xi_{0}\right), A\left(\xi_{0}\right)=D f\left(\mathbf{0}, \xi_{0}\right)$, has eigenvalues $\lambda$ and $\bar{\lambda}$, where $\lambda\left(\xi_{0}\right)=\gamma\left(\xi_{0}\right)+i \eta\left(\xi_{0}\right), \gamma\left(\xi_{0}\right)=0$ and $\eta\left(\xi_{0}\right)=\omega_{0}(\nu)>0$. Let also $q(\xi) \in \mathbb{C}^{2}$ be an eigenvector corresponding to the eigenvalue $\lambda(\xi)$, and let $p(\xi) \in \mathbb{C}^{2}$ be an adjoint eigenvector corresponding to the eigenvalue $\bar{\lambda}(\xi)$, satisfying (6), (7), and (8). The following statements hold.

(a) The vector $\partial_{\mu} q\left(\xi_{0}\right) \in \mathbb{C}^{2}$ is the solution of the following nonsingular 3-dimensional system:

$$
\left(\begin{array}{cc}
i \omega_{0}(\nu) I_{2}-A\left(\xi_{0}\right) & q\left(\xi_{0}\right) \\
\bar{p}\left(\xi_{0}\right) & 0
\end{array}\right)\left(\begin{array}{c}
\partial_{\mu} q\left(\xi_{0}\right) \\
s
\end{array}\right)=\left(\begin{array}{c}
R_{2}\left(\xi_{0}\right) \\
0
\end{array}\right),
$$

with the condition $\left\langle p\left(\xi_{0}\right), \partial_{\mu} q\left(\xi_{0}\right)\right\rangle=0$, where

$$
R_{2}\left(\xi_{0}\right)=\left(\partial_{\mu} A\left(\xi_{0}\right)-\partial_{\mu} \lambda\left(\xi_{0}\right) I_{2}\right) q\left(\xi_{0}\right) .
$$

(b) The vector $\partial_{\mu} p\left(\xi_{0}\right) \in \mathbb{C}^{2}$ is the solution of the following nonsingular 3-dimensional system:

$$
\left(\begin{array}{cc}
-\left(i \omega_{0}(\nu) I_{2}+A^{T}\left(\xi_{0}\right)\right) & p\left(\xi_{0}\right) \\
\bar{q}\left(\xi_{0}\right) & 0
\end{array}\right)\left(\begin{array}{c}
\partial_{\mu} p\left(\xi_{0}\right) \\
s
\end{array}\right)=\left(\begin{array}{c}
\bar{R}_{2}\left(\xi_{0}\right) \\
0
\end{array}\right)
$$

with the condition $\left\langle q\left(\xi_{0}\right), \partial_{\mu} p\left(\xi_{0}\right)\right\rangle=0$, where

$$
\bar{R}_{2}\left(\xi_{0}\right)=\left(\partial_{\mu} A^{T}\left(\xi_{0}\right)-\partial_{\mu} \bar{\lambda}\left(\xi_{0}\right) I_{2}\right) p\left(\xi_{0}\right) .
$$

(c) The partial derivative with respect to $\mu$ of the real part of the eigenvalue $\lambda(\xi)$, evaluated at $\xi=\xi_{0}$, is given by

$$
\partial_{\mu} \gamma\left(\xi_{0}\right)=\operatorname{Re}\left(\left\langle p\left(\xi_{0}\right), \partial_{\mu} A\left(\xi_{0}\right) q\left(\xi_{0}\right)\right\rangle\right) .
$$

(d) The partial derivative with respect to $\mu$ of the imaginary part of the eigenvalue $\lambda(\xi)$, evaluated at $\xi=\xi_{0}$, is given by

$$
\partial_{\mu} \eta\left(\xi_{0}\right)=\operatorname{Im}\left(\left\langle p\left(\xi_{0}\right), \partial_{\mu} A\left(\xi_{0}\right) q\left(\xi_{0}\right)\right\rangle\right) .
$$

(e) The second-order partial derivative with respect to $\mu$ of the real part of the eigenvalue $\lambda(\xi)$, evaluated at $\xi=\xi_{0}$, is given by

$$
\begin{aligned}
\partial_{\mu}^{2} \gamma\left(\xi_{0}\right)=\operatorname{Re}( & \left\langle p\left(\xi_{0}\right), \partial_{\mu}^{2} A\left(\xi_{0}\right) q\left(\xi_{0}\right)\right. \\
& \left.\left.+2\left(\partial_{\mu} A\left(\xi_{0}\right)-\partial_{\mu} \lambda\left(\xi_{0}\right) I_{2}\right) \partial_{\mu} q\left(\xi_{0}\right)\right\rangle\right) .
\end{aligned}
$$

(f) The second-order partial derivative with respect to $\mu$ of the imaginary part of the eigenvalue $\lambda(\xi)$, evaluated at $\xi=\xi_{0}$, is given by

$$
\begin{aligned}
\partial_{\mu}^{2} \eta\left(\xi_{0}\right)=\operatorname{Im} & \left(\left\langlep\left(\xi_{0}\right), \partial_{\mu}^{2} A\left(\xi_{0}\right) q\left(\xi_{0}\right)\right.\right. \\
& \left.\left.+2\left(\partial_{\mu} A\left(\xi_{0}\right)-\partial_{\mu} \lambda\left(\xi_{0}\right) I_{2}\right) \partial_{\mu} q\left(\xi_{0}\right)\right\rangle\right) .
\end{aligned}
$$


Proof. Differentiating (6) with respect to the parameter $\mu$ and evaluating at $\xi=\xi_{0}$, we have

$$
\begin{aligned}
\partial_{\mu} A & \left(\xi_{0}\right) q\left(\xi_{0}\right)+A\left(\xi_{0}\right) \partial_{\mu} q\left(\xi_{0}\right) \\
& =\partial_{\mu} \lambda\left(\xi_{0}\right) q\left(\xi_{0}\right)+\lambda\left(\xi_{0}\right) \partial_{\mu} q\left(\xi_{0}\right) .
\end{aligned}
$$

Using the hypotheses, the previous equation is rewritten as

$$
\left(i \omega_{0}(\nu) I_{2}-A\left(\xi_{0}\right)\right) \partial_{\mu} q\left(\xi_{0}\right)=\left(\partial_{\mu} A\left(\xi_{0}\right)-\partial_{\mu} \lambda\left(\xi_{0}\right)\right) q\left(\xi_{0}\right) .
$$

Taking the inner product of $p\left(\xi_{0}\right) \in \mathbb{C}^{2}$ on both sides of the above equation and using (8), it follows that

$$
\begin{aligned}
0 & =\left\langle p\left(\xi_{0}\right),\left(i \omega_{0}(\nu) I_{2}-A\left(\xi_{0}\right)\right) \partial_{\mu} q\left(\xi_{0}\right)\right\rangle \\
& =\left\langle p\left(\xi_{0}\right), \partial_{\mu} A\left(\xi_{0}\right) q\left(\xi_{0}\right)\right\rangle-\partial_{\mu} \lambda\left(\xi_{0}\right) .
\end{aligned}
$$

Items (a), (c), and (d) follow from the above equation, the Fredholm alternative (see [1]), and the results of [8]. The proof of part (b) is equal to the previous proof; that is, it is sufficient to differentiate (7) with respect to the parameter $\mu$ and to evaluate at $\xi=\xi_{0}$. The proofs of items (e) and (f) consist of calculating the second-order partial derivative of (6) with respect to the parameter $\mu$, evaluated at $\xi=\xi_{0}$, and to use the Fredholm alternative.

Proposition 8. Consider the coefficients of the formal Taylor series of the map $(z, \bar{z}, \xi) \mapsto g(z, \bar{z}, \xi)$,

$$
\begin{aligned}
& g_{2,0}(\xi)=\langle p(\xi), B(q(\xi), q(\xi), \xi)\rangle, \\
& g_{1,1}(\xi)=\langle p(\xi), B(q(\xi), \bar{q}(\xi), \xi)\rangle, \\
& g_{0,2}(\xi)=\langle p(\xi), B(\bar{q}(\xi), \bar{q}(\xi), \xi)\rangle, \\
& g_{2,1}(\xi)=\langle p(\xi), C(q(\xi), q(\xi), \bar{q}(\xi), \xi)\rangle .
\end{aligned}
$$

The following statements hold.

(a) The partial derivative with respect to $\mu$ of the coefficient $g_{2,0}(\xi)$, evaluated at $\xi=\xi_{0}$, is

$$
\begin{aligned}
\partial_{\mu} g_{2,0}\left(\xi_{0}\right)= & \left\langle\partial_{\mu} p\left(\xi_{0}\right), B\left(q\left(\xi_{0}\right), q\left(\xi_{0}\right), \xi_{0}\right)\right\rangle \\
+ & \left\langle p\left(\xi_{0}\right), 2 B\left(q\left(\xi_{0}\right), \partial_{\mu} q\left(\xi_{0}\right), \xi_{0}\right)\right. \\
& \left.+\partial_{\mu} B\left(q\left(\xi_{0}\right), q\left(\xi_{0}\right), \xi_{0}\right)\right\rangle .
\end{aligned}
$$

(b) The partial derivative with respect to $\mu$ of the coefficient $g_{1,1}(\xi)$, evaluated at $\xi=\xi_{0}$, is given by

$$
\begin{aligned}
\partial_{\mu} g_{1,1}\left(\xi_{0}\right)= & \left\langle\partial_{\mu} p\left(\xi_{0}\right), B\left(q\left(\xi_{0}\right), \bar{q}\left(\xi_{0}\right), \xi_{0}\right)\right\rangle \\
+ & \left\langle p\left(\xi_{0}\right), B\left(\partial_{\mu} q\left(\xi_{0}\right), \bar{q}\left(\xi_{0}\right), \xi_{0}\right)\right. \\
& +B\left(q\left(\xi_{0}\right), \partial_{\mu} \bar{q}\left(\xi_{0}\right), \xi_{0}\right) \\
& \left.+\partial_{\mu} B\left(q\left(\xi_{0}\right), \bar{q}\left(\xi_{0}\right), \xi_{0}\right)\right\rangle .
\end{aligned}
$$

(c) The partial derivative with respect to $\mu$ of the coefficient $g_{0,2}(\xi)$, evaluated at $\xi=\xi_{0}$, is obtained as

$$
\begin{aligned}
\partial_{\mu} g_{0,2}\left(\xi_{0}\right)= & \left\langle\partial_{\mu} p\left(\xi_{0}\right), B\left(\bar{q}\left(\xi_{0}\right), \bar{q}\left(\xi_{0}\right), \xi_{0}\right)\right\rangle \\
+ & \left\langle p\left(\xi_{0}\right), 2 B\left(\bar{q}\left(\xi_{0}\right), \partial_{\mu} \bar{q}\left(\xi_{0}\right), \xi_{0}\right)\right. \\
& \left.+\partial_{\mu} B\left(\bar{q}\left(\xi_{0}\right), \bar{q}\left(\xi_{0}\right), \xi_{0}\right)\right\rangle .
\end{aligned}
$$

(d) The partial derivative with respect to $\mu$ of the coefficient $g_{2,1}(\xi)$, evaluated at $\xi=\xi_{0}$, is calculated as

$$
\begin{aligned}
\partial_{\mu} g_{2,1}\left(\xi_{0}\right)= & \left\langle\partial_{\mu} p\left(\xi_{0}\right), C\left(q\left(\xi_{0}\right), q\left(\xi_{0}\right), \bar{q}\left(\xi_{0}\right), \xi_{0}\right)\right\rangle \\
+ & \left\langle p\left(\xi_{0}\right), 2 C\left(q\left(\xi_{0}\right), \partial_{\mu} q\left(\xi_{0}\right), \bar{q}\left(\xi_{0}\right), \xi_{0}\right)\right. \\
& +C\left(q\left(\xi_{0}\right), q\left(\xi_{0}\right), \partial_{\mu} \bar{q}\left(\xi_{0}\right), \xi_{0}\right) \\
& \left.+\partial_{\mu} C\left(q\left(\xi_{0}\right), q\left(\xi_{0}\right), \bar{q}\left(\xi_{0}\right), \xi_{0}\right)\right\rangle .
\end{aligned}
$$

Proof. Observing how the symmetric multilinear functions are defined, the proofs of items (a) to (d) consist in differentiating each expression in (81) with respect to the parameter $\mu$ and evaluating at $\xi=\xi_{0}$.

The theory built up to this point approximates a family of periodic orbits of the complex differential equation (11). In the hypotheses of the Hopf bifurcation, if $(\omega(\epsilon, \nu) t, \epsilon, \nu) \mapsto$ $w(\omega(\epsilon, \nu) t, \epsilon, \nu)$ is a family of periodic orbits of (11), then $(\omega(\epsilon, \nu) t, \epsilon, \nu) \mapsto u(\omega(\epsilon, \nu) t, \epsilon, \nu)$ is a family of periodic orbits associated with the differential equation (1), where

$$
\begin{aligned}
u(\omega(\epsilon, \nu) t, \epsilon, \nu)= & w(\omega(\epsilon, \nu) t, \epsilon, \nu) q(\phi(\epsilon, \nu), \nu) \\
& +\bar{w}(\omega(\epsilon, \nu) t, \epsilon, \nu) \bar{q}(\phi(\epsilon, \nu), \nu)
\end{aligned}
$$

or, in a more simple way,

$u(s, \epsilon, \nu)=w(s, \epsilon, \nu) q(\phi(\epsilon, \nu), \nu)+\bar{w}(s, \epsilon, \nu) \bar{q}(\phi(\epsilon, \nu), \nu)$

The family of periodic orbits $(s, \epsilon, \nu) \mapsto u(s, \epsilon, \nu)$ has formal Taylor series around $\epsilon=0$ of the following form:

$$
u(s, \epsilon, \nu)=\sum_{k=1}^{\infty} \frac{1}{k !} u_{k}(s, \nu) \epsilon^{k}
$$

and the theory developed previously and the Taylor expansion of (87), around $\epsilon=0$, show that

$$
\begin{array}{r}
u_{1}(s, v)=q\left(\xi_{0}\right) w_{1}(s, v)+\bar{q}\left(\xi_{0}\right) \bar{w}_{1}(s, \nu), \\
u_{2}(s, v)=q\left(\xi_{0}\right) w_{2}(s, \nu)+\bar{q}\left(\xi_{0}\right) \bar{w}_{2}(s, \nu), \\
u_{3}(s, \nu)=q\left(\xi_{0}\right) w_{3}(s, \nu)+\bar{q}\left(\xi_{0}\right) \bar{w}_{3}(s, \nu) \\
+3 \mu_{2}(\nu)\left(w_{1}(s, \nu) \partial_{\mu} q\left(\xi_{0}\right)\right. \\
\left.+\bar{w}_{1}(s, \nu) \partial_{\mu} \bar{q}\left(\xi_{0}\right)\right),
\end{array}
$$




$$
\begin{aligned}
u_{4}(s, \nu)= & q\left(\xi_{0}\right) w_{4}(s, \nu)+\bar{q}\left(\xi_{0}\right) \bar{w}_{4}(s, v) \\
& +6 \mu_{2}(\nu)\left(w_{2}(s, \nu) \partial_{\mu} q\left(\xi_{0}\right)\right. \\
& \left.+\bar{w}_{2}(s, \nu) \partial_{\mu} \bar{q}\left(\xi_{0}\right)\right) .
\end{aligned}
$$

The stability of the approximate family of periodic orbits is studied in the next section by means of the Floquet exponent.

\section{Stability of the Family of Periodic Orbits}

According to the Floquet theory (see [9]), the stability of a periodic orbit can be determined through the characteristic exponent that, in this context and for differential equations in $\mathbb{R}^{2}$, is a function $(\epsilon, \nu) \mapsto \chi(\epsilon, \nu)$ such that

$$
\chi(\epsilon, \nu)=\frac{1}{T(\epsilon, \nu)} \int_{0}^{T(\epsilon, \nu)} \operatorname{Tr}(\mathscr{M}(\omega(\epsilon, \nu) t, \epsilon, \nu)) d t
$$

where $\mathscr{M}(\omega(\epsilon, \nu) t, \epsilon, \nu)=D f(u(\omega(\epsilon, \nu) t, \epsilon, \nu), \phi(\epsilon, \nu), \nu)$. The next proposition provides a simple way to compute (90) in terms of the map $(z, \bar{z}, \xi) \mapsto g(z, \bar{z}, \xi)$

Proposition 9. Through a change in time $s=\omega(\epsilon, \nu) t$, the characteristic exponent associated with the differential equation $z^{\prime}=g(z, \bar{z}, \xi)$ is of the following form:

$$
\chi(\epsilon, \nu)=\frac{1}{2 \pi} \int_{0}^{2 \pi} \mathscr{H}(s, \epsilon, \nu) d s
$$

where

$$
\begin{aligned}
\mathscr{H}(s, \epsilon, \nu)= & \frac{\partial}{\partial w} g(w(s, \epsilon, \nu), \bar{w}(s, \epsilon, \nu), \phi(\epsilon, \nu), \nu) \\
& +\frac{\partial}{\partial \bar{w}} \bar{g}(w(s, \epsilon, \nu), \bar{w}(s, \epsilon, \nu), \phi(\epsilon, \nu), \nu) .
\end{aligned}
$$

Proof. The differential equation (1) can be written as (11), where $(z, \bar{z}, \xi) \mapsto g(z, \bar{z}, \xi)=g_{1}\left(z_{1}, z_{2}, \xi\right)+i g_{2}\left(z_{1}, z_{2}, \xi\right)$,

$$
\begin{gathered}
g_{1}\left(w_{1}, w_{2}, \xi\right)=\frac{1}{2}(g(w, \bar{w}, \xi)+\bar{g}(w, \bar{w}, \xi)), \\
g_{2}\left(w_{1}, w_{2}, \xi\right)=-\frac{i}{2}(g(w, \bar{w}, \xi)-\bar{g}(w, \bar{w}, \xi)),
\end{gathered}
$$

and $z=z_{1}+i z_{2}$. Thus, through the changes $z(t)=w(s, \epsilon, \nu)$ and $s=\omega(\epsilon, \nu) t$, the characteristic exponent (90) can be rewritten as

$$
\begin{aligned}
\chi & (\epsilon, \nu) \\
& =\frac{1}{2 \pi} \int_{0}^{2 \pi}\left(\frac{\partial}{\partial w_{1}} g_{1}\left(w_{1}, w_{2}, \xi\right)+\frac{\partial}{\partial w_{2}} g_{2}\left(w_{1}, w_{2}, \xi\right)\right) d s,
\end{aligned}
$$

where

$$
\begin{aligned}
& \frac{\partial}{\partial w_{1}} g_{1}\left(w_{1}, w_{2}, \xi\right) \\
& =\frac{1}{2}\left(\frac{\partial}{\partial w} g(w, \bar{w}, \xi) \frac{\partial w}{\partial w_{1}}+\frac{\partial}{\partial \bar{w}} g(w, \bar{w}, \xi) \frac{\partial \bar{w}}{\partial w_{1}}\right) \\
& \quad+\frac{1}{2}\left(\frac{\partial}{\partial w} \bar{g}(w, \bar{w}, \xi) \frac{\partial w}{\partial w_{1}}+\frac{\partial}{\partial \bar{w}} \bar{g}(w, \bar{w}, \xi) \frac{\partial \bar{w}}{\partial w_{1}}\right), \\
& \frac{\partial}{\partial w_{2}} g_{2}\left(w_{1}, w_{2}, \xi\right) \\
& =-\frac{i}{2}\left(\frac{\partial}{\partial w} g(w, \bar{w}, \xi) \frac{\partial w}{\partial w_{2}}+\frac{\partial}{\partial \bar{w}} g(w, \bar{w}, \xi) \frac{\partial \bar{w}}{\partial w_{2}}\right) \\
& \quad+\frac{i}{2}\left(\frac{\partial}{\partial w} \bar{g}(w, \bar{w}, \xi) \frac{\partial w}{\partial w_{2}}+\frac{\partial}{\partial \bar{w}} \bar{g}(w, \bar{w}, \xi) \frac{\partial \bar{w}}{\partial w_{2}}\right) .
\end{aligned}
$$

Adding equations (95) and (96) and taking into account that $w=w_{1}+i w_{2}$, it follows that

$$
\begin{gathered}
\frac{\partial}{\partial w_{1}} g_{1}\left(w_{1}, w_{2}, \xi\right)+\frac{\partial}{\partial w_{2}} g_{2}\left(w_{1}, w_{2}, \xi\right) \\
=\frac{\partial}{\partial w} g(w, \bar{w}, \xi)+\frac{\partial}{\partial \bar{w}} \bar{g}(w, \bar{w}, \xi) .
\end{gathered}
$$

Therefore, $\mathscr{H}(s, \epsilon, v)=K(w, \bar{w}, \xi)$, with

$$
K(w, \bar{w}, \xi)=\frac{\partial}{\partial w} g(w, \bar{w}, \xi)+\frac{\partial}{\partial \bar{w}} \bar{g}(w, \bar{w}, \xi) .
$$

By the formal Taylor series in the variable $\epsilon$ of the function $(\epsilon, \nu) \mapsto \chi(\epsilon, \nu)$,

$$
\chi(\epsilon, \nu)=\sum_{k=1}^{\infty} \frac{1}{k !} \chi_{k}(\nu) \epsilon^{k}
$$

the theory of approximation of a family of periodic orbits developed in the previous section and Proposition 9 allow us to obtain the terms of the sequence $\left\{\chi_{k}(\nu)\right\}_{k \in \mathbb{N}}$. For $k=$ $1, \ldots, 4$, the next theorem provides these terms.

Theorem 10. Let

$$
\chi(\epsilon, \nu)=\sum_{k=1}^{\infty} \frac{1}{k !} \chi_{k}(\nu) \epsilon^{k}
$$

be the formal Taylor series of the characteristic exponent $(\epsilon, \nu) \mapsto \chi(\epsilon, \nu)$ associated with the differential equation $z^{\prime}=$ $g(z, \bar{z}, \xi)$. Then,

$$
\begin{aligned}
& \chi_{1}(\nu)=0, \\
& \chi_{2}(\nu)=2 \operatorname{Re}\left(G_{2,1}\left(\xi_{0}\right)\right), \\
& \chi_{3}(\nu)=0, \\
& \chi_{4}(\nu)=8 \operatorname{Re}\left(G_{3,2}\left(\xi_{0}\right)\right)+12 \mu_{2}(\nu) \operatorname{Re}\left(\partial_{\mu} G_{2,1}\left(\xi_{0}\right)\right), \\
& \chi_{5}(\nu)=0,
\end{aligned}
$$


where $G_{2,1}\left(\xi_{0}\right), \partial_{\mu} G_{2,1}\left(\xi_{0}\right)$, and $G_{3,2}\left(\xi_{0}\right)$ are given by (50), (63), and (64), respectively.

Proof. From (13) and (14), we have

$$
\begin{aligned}
& \frac{\partial}{\partial w} g(w, \bar{w}, \xi)=\lambda(\xi)+\sum_{k=2}^{\infty} \sum_{j=0}^{k} \frac{k-j}{(k-j) ! j !} g_{k-j, j}(\xi) w^{k-j-1} \bar{w}^{j} \\
& \frac{\partial}{\partial \bar{w}} \bar{g}(w, \bar{w}, \xi)=\bar{\lambda}(\xi)+\sum_{k=2}^{\infty} \sum_{j=0}^{k} \frac{k-j}{(k-j) ! j !} \bar{g}_{k-j, j}(\xi) \bar{w}^{k-j-1} w^{j}
\end{aligned}
$$

Thus, formally, the map $(w, \bar{w}, \xi) \mapsto K(w, \bar{w}, \xi)$ has the Taylor series

$$
\begin{aligned}
K(w, \bar{w}, \xi)= & \lambda(\xi)+\bar{\lambda}(\xi) \\
+ & +\sum_{k=2}^{\infty} \sum_{j=0}^{k} \frac{k-j}{(k-j) ! j !} \\
& \quad \times\left(g_{k-j, j}(\xi)+\bar{g}_{k-j, j}(\xi)\right) w^{k-j-1} \bar{w}^{j}
\end{aligned}
$$

Doing the fourth-order Taylor expansion of the map

$$
(s, \epsilon, \nu) \mapsto \mathscr{H}(s, \epsilon, \nu)=K(w(s, \epsilon, \nu), \bar{w}(s, \epsilon, \nu), \phi(\epsilon, \nu), \nu),
$$

around $\epsilon=0$, and taking into account that $\mu_{1}(\nu)=\mu_{3}(\nu)=0$, it results that

$$
\begin{aligned}
\mathscr{H}(s, \epsilon, v)= & \mathscr{H}_{1}(s, \nu) \epsilon+\frac{1}{2} \mathscr{H}_{2}(s, \nu) \epsilon^{2} \\
& +\frac{1}{6} \mathscr{H}_{3}(s, v) \epsilon^{3}+\frac{1}{24} \mathscr{H}_{4}(s, v) \epsilon^{4} \\
& +O_{\mathscr{H}}\left(s, \epsilon^{5},|v|\right),
\end{aligned}
$$

with

$$
\begin{aligned}
\mathscr{H}_{1}(s, v)= & \bar{w}_{1}(s, v) g_{1,1}\left(\xi_{0}\right)+w_{1}(s, v) g_{2,0}\left(\xi_{0}\right) \\
& +w_{1}(s, v) \bar{g}_{1,1}\left(\xi_{0}\right)+\bar{w}_{1}(s, v) \bar{g}_{2,0}\left(\xi_{0}\right) \\
\mathscr{H}_{2}(s, v)= & w_{1}(s, v)^{2} g_{3,0}\left(\xi_{0}\right)+w_{1}(s, v)^{2} \bar{g}_{1,2}\left(\xi_{0}\right) \\
& +2 w_{1}(s, v) \bar{w}_{1}(s, v) g_{2,1}\left(\xi_{0}\right) \\
& +2 w_{1}(s, v) \bar{w}_{1}(s, v) \bar{g}_{2,1}\left(\xi_{0}\right) \\
& +\bar{w}_{2}(s, v) g_{1,1}\left(\xi_{0}\right)+\bar{w}_{1}(s, v)^{2} g_{1,2}\left(\xi_{0}\right) \\
& +w_{2}(s, v) g_{2,0}\left(\xi_{0}\right)+w_{2}(s, v) \bar{g}_{1,1}\left(\xi_{0}\right) \\
& +\bar{w}_{2}(s, v) \bar{g}_{2,0}\left(\xi_{0}\right) \\
& +\bar{w}_{1}(s, v)^{2} \bar{g}_{3,0}\left(\xi_{0}\right)+2 \mu_{2}(\nu) \partial_{\mu} \gamma\left(\xi_{0}\right) \\
\mathscr{H}_{3}(s, v)= & w_{1}(s, v)^{3} g_{4,0}\left(\xi_{0}\right)+w_{1}(s, v)^{3} \bar{g}_{1,3}\left(\xi_{0}\right) \\
& +3 w_{1}(s, v)^{2} \bar{w}_{1}(s, v) g_{3,1}\left(\xi_{0}\right) \\
& +3 w_{1}(s, v)^{2} \bar{w}_{1}(s, v) \bar{g}_{2,2}\left(\xi_{0}\right)
\end{aligned}
$$

$$
\begin{aligned}
& +3 w_{1}(s, v) \bar{w}_{2}(s, v) g_{2,1}\left(\xi_{0}\right) \\
& +3 w_{1}(s, v) \bar{w}_{1}(s, v)^{2} g_{2,2}\left(\xi_{0}\right) \\
& +3 w_{1}(s, \nu) w_{2}(s, \nu) g_{3,0}\left(\xi_{0}\right) \\
& +3 w_{1}(s, v) w_{2}(s, v) \bar{g}_{1,2}\left(\xi_{0}\right) \\
& +3 w_{1}(s, v) \bar{w}_{2}(s, v) \bar{g}_{2,1}\left(\xi_{0}\right) \\
& +3 w_{1}(s, v) \bar{w}_{1}(s, v)^{2} \bar{g}_{3,1}\left(\xi_{0}\right) \\
& +3 \mu_{2}(\nu) w_{1}(s, \nu) \partial_{\mu} g_{2,0}\left(\xi_{0}\right) \\
& +3 \mu_{2}(\nu) \partial_{\mu} \bar{g}_{1,1}\left(\xi_{0}\right) w_{1}(s, v) \\
& +\bar{w}_{3}(s, \nu) g_{1,1}\left(\xi_{0}\right) \\
& +3 \bar{w}_{1}(s, \nu) \bar{w}_{2}(s, \nu) g_{1,2}\left(\xi_{0}\right) \\
& +\bar{w}_{1}(s, v)^{3} g_{1,3}\left(\xi_{0}\right)+w_{3}(s, v) g_{2,0}\left(\xi_{0}\right) \\
& +3 w_{2}(s, v) \bar{w}_{1}(s, \nu) g_{2,1}\left(\xi_{0}\right) \\
& +w_{3}(s, \nu) \bar{g}_{1,1}\left(\xi_{0}\right)+\bar{w}_{3}(s, \nu) \bar{g}_{2,0}\left(\xi_{0}\right) \\
& +3 w_{2}(s, v) \bar{w}_{1}(s, v) \bar{g}_{2,1}\left(\xi_{0}\right) \\
& +3 \bar{w}_{1}(s, v) \bar{w}_{2}(s, v) \bar{g}_{3,0}\left(\xi_{0}\right) \\
& +\bar{w}_{1}(s, v)^{3} \bar{g}_{4,0}\left(\xi_{0}\right)+3 \mu_{2}(v) \bar{w}_{1}(s, v) \partial_{\mu} g_{1,1}\left(\xi_{0}\right) \\
& +3 \mu_{2}(\nu) \bar{w}_{1}(s, \nu) \partial_{\mu} \bar{g}_{2,0}\left(\xi_{0}\right), \\
& \mathscr{H}_{4}(s, v)=w_{1}(s, v)^{4} g_{5,0}\left(\xi_{0}\right)+w_{1}(s, v)^{4} \bar{g}_{1,4}\left(\xi_{0}\right) \\
& +4 w_{1}(s, \nu)^{3} \bar{w}_{1}(s, \nu) g_{4,1}\left(\xi_{0}\right) \\
& +4 w_{1}(s, v)^{3} \bar{w}_{1}(s, v) \bar{g}_{2,3}\left(\xi_{0}\right) \\
& +6 w_{1}(s, v)^{2} \bar{w}_{2}(s, v) g_{3,1}\left(\xi_{0}\right) \\
& +6 w_{1}(s, v)^{2} \bar{w}_{1}(s, v)^{2} g_{3,2}\left(\xi_{0}\right) \\
& +6 w_{1}(s, v)^{2} w_{2}(s, v) g_{4,0}\left(\xi_{0}\right) \\
& +6 w_{1}(s, v)^{2} w_{2}(s, v) \bar{g}_{1,3}\left(\xi_{0}\right) \\
& +6 w_{1}(s, v)^{2} \bar{w}_{2}(s, v) \bar{g}_{2,2}\left(\xi_{0}\right) \\
& +6 w_{1}(s, v)^{2} \bar{w}_{1}(s, v)^{2} \bar{g}_{3,2}\left(\xi_{0}\right) \\
& +6 \mu_{2}(\nu) w_{1}(s, \nu)^{2} \partial_{\mu} g_{3,0}\left(\xi_{0}\right) \\
& +6 \mu_{2}(\nu) w_{1}(s, v)^{2} \partial_{\mu} \bar{g}_{1,2}\left(\xi_{0}\right) \\
& +4 w_{1}(s, v) \bar{w}_{3}(s, v) g_{2,1}\left(\xi_{0}\right) \\
& +12 w_{1}(s, v) \bar{w}_{1}(s, v) \bar{w}_{2}(s, v) g_{2,2}\left(\xi_{0}\right) \\
& +4 w_{1}(s, v) \bar{w}_{1}(s, v)^{3} g_{2,3}\left(\xi_{0}\right) \\
& +12 w_{1}(s, \nu) \bar{w}_{1}(s, \nu) w_{2}(s, \nu) g_{3,1}\left(\xi_{0}\right)
\end{aligned}
$$




$$
\begin{aligned}
& +4 w_{1}(s, v) w_{3}(s, \nu) g_{3,0}\left(\xi_{0}\right) \\
& +4 w_{1}(s, \nu) w_{3}(s, \nu) \bar{g}_{1,2}\left(\xi_{0}\right) \\
& +4 w_{1}(s, v) \bar{w}_{3}(s, \nu) \bar{g}_{2,1}\left(\xi_{0}\right) \\
& +12 w_{1}(s, \nu) \bar{w}_{1}(s, \nu) w_{2}(s, \nu) \bar{g}_{2,2}\left(\xi_{0}\right) \\
& +12 w_{1}(s, \nu) \bar{w}_{1}(s, v) \bar{w}_{2}(s, \nu) \bar{g}_{3,1}\left(\xi_{0}\right) \\
& +4 w_{1}(s, v) \bar{w}_{1}(s, v)^{3} \bar{g}_{4,1}\left(\xi_{0}\right) \\
& +12 \mu_{2}(\nu) w_{1}(s, \nu) \bar{w}_{1}(s, \nu) \partial_{\mu} g_{2,1}\left(\xi_{0}\right) \\
& +12 \mu_{2}(\nu) w_{1}(s, \nu) \bar{w}_{1}(s, \nu) \partial_{\mu} \bar{g}_{2,1}\left(\xi_{0}\right) \\
& +\bar{w}_{4}(s, \nu) g_{1,1}\left(\xi_{0}\right)+3 \bar{w}_{2}(s, \nu)^{2} g_{1,2}\left(\xi_{0}\right) \\
& +4 \bar{w}_{1}(s, \nu) \bar{w}_{3}(s, \nu) g_{1,2}\left(\xi_{0}\right) \\
& +6 \bar{w}_{1}(s, v)^{2} \bar{w}_{2}(s, v) g_{1,3}\left(\xi_{0}\right) \\
& +\bar{w}_{1}(s, v)^{4} g_{1,4}\left(\xi_{0}\right) \\
& +w_{4}(s, \nu) g_{2,0}\left(\xi_{0}\right) \\
& +4 \bar{w}_{1}(s, v) w_{3}(s, v) g_{2,1}\left(\xi_{0}\right) \\
& +6 w_{2}(s, v) \bar{w}_{2}(s, \nu) g_{2,1}\left(\xi_{0}\right) \\
& +6 \bar{w}_{1}(s, v)^{2} w_{2}(s, v) g_{2,2}\left(\xi_{0}\right) \\
& +3 w_{2}(s, v)^{2} g_{3,0}\left(\xi_{0}\right) \\
& +w_{4}(s, v) \bar{g}_{1,1}\left(\xi_{0}\right) \\
& +3 w_{2}(s, v)^{2} \bar{g}_{1,2}\left(\xi_{0}\right) \\
& +\bar{w}_{4}(s, v) \bar{g}_{2,0}\left(\xi_{0}\right) \\
& +4 \bar{w}_{1}(s, \nu) w_{3}(s, \nu) \bar{g}_{2,1}\left(\xi_{0}\right) \\
& +6 w_{2}(s, v) \bar{w}_{2}(s, v) \bar{g}_{2,1}\left(\xi_{0}\right) \\
& +3 \bar{w}_{2}(s, v)^{2} \bar{g}_{3,0}\left(\xi_{0}\right) \\
& +4 \bar{w}_{1}(s, v) \bar{w}_{3}(s, v) \bar{g}_{3,0}\left(\xi_{0}\right) \\
& +6 \bar{w}_{1}(s, v)^{2} w_{2}(s, v) \bar{g}_{3,1}\left(\xi_{0}\right) \\
& +6 \bar{w}_{1}(s, v)^{2} \bar{w}_{2}(s, v) \bar{g}_{4,0}\left(\xi_{0}\right) \\
& +\bar{w}_{1}(s, \nu)^{4} \bar{g}_{5,0}\left(\xi_{0}\right) \\
& +6 \mu_{2}(\nu) \bar{w}_{2}(s, \nu) \partial_{\mu} g_{1,1}\left(\xi_{0}\right) \\
& +2 \mu_{4}(\nu) \partial_{\mu} \gamma\left(\xi_{0}\right) \\
& +6 \mu_{2}(\nu) \bar{w}_{1}(s, \nu)^{2} \partial_{\mu} g_{1,2}\left(\xi_{0}\right) \\
& +6 \mu_{2}(\nu) w_{2}(s, \nu) \partial_{\mu} g_{2,0}\left(\xi_{0}\right) \\
& +6 \mu_{2}(\nu) w_{2}(s, v) \partial_{\mu} \bar{g}_{1,1}\left(\xi_{0}\right)
\end{aligned}
$$

$$
\begin{aligned}
& +6 \mu_{2}(\nu) \bar{w}_{2}(s, \nu) \partial_{\mu} \bar{g}_{2,0}\left(\xi_{0}\right) \\
& +6 \mu_{2}(\nu) \bar{w}_{1}(s, \nu)^{2} \partial_{\mu} \bar{g}_{3,0}\left(\xi_{0}\right) \\
& +6 \mu_{2}(\nu)^{2} \partial_{\mu}^{2} \gamma\left(\xi_{0}\right)
\end{aligned}
$$

where for $k=1,2,3,4$, the functions $(s, \nu) \mapsto w_{k}(s, \nu)$ are such as in (37), (46), (54), and (59) and the expressions $\mu_{2}(\nu)$ and $\mu_{4}(\nu)$ are given by (52), and (61), respectively. Thus, from (99) and (105) and by Proposition 9,

$$
\begin{aligned}
\sum_{k=1}^{4} & \frac{1}{k !}\left(\chi_{k}(\epsilon, \nu)-\frac{1}{2 \pi} \int_{0}^{2 \pi} \mathscr{H}_{k}(s, \nu) d s\right) \epsilon^{k} \\
& +O_{\mathscr{H}_{I}}\left(\epsilon^{5},|\nu|\right)=0 .
\end{aligned}
$$

Therefore,

$$
\begin{aligned}
& \frac{1}{2 \pi} \int_{0}^{2 \pi} \mathscr{H}_{1}(s, v) d s=\frac{1}{2 \pi} \int_{0}^{2 \pi} \mathscr{H}_{3}(s, v) d s=0, \\
& \frac{1}{2 \pi} \int_{0}^{2 \pi} \mathscr{H}_{2}(s, v) d s=2 \operatorname{Re}\left(G_{2,1}\left(\xi_{0}\right)\right), \\
& \frac{1}{2 \pi} \int_{0}^{2 \pi} \mathscr{H}_{4}(s, \nu) d s= 8 \operatorname{Re}\left(G_{3,2}\left(\xi_{0}\right)\right) \\
&+12 \mu_{2}(\nu) \operatorname{Re}\left(\partial_{\mu} G_{2,1}\left(\xi_{0}\right)\right),
\end{aligned}
$$

which proves the theorem.

It follows from Theorem 10 a corollary that deals with the stability of a family of periodic orbits of the differential equation (1) which exists due to a Hopf bifurcation.

Corollary 11. Let

$$
\chi(\epsilon, \nu)=\frac{1}{2} \chi_{2}(\nu) \epsilon^{2}+\frac{1}{24} \chi_{4}(\nu) \epsilon^{4}+O_{\chi}\left(\epsilon^{5},|\nu|\right)
$$

be the fourth-order Taylor expansion around $\epsilon=0$ of the characteristic exponent $(\epsilon, \nu) \mapsto \chi(\epsilon, \nu)$ associated with the differential equation $z^{\prime}=g(z, \bar{z}, \xi)$, and let

$$
\mu=\phi(\epsilon, \nu)=\frac{1}{2} \mu_{2}(\nu) \epsilon^{2}+\frac{1}{24} \mu_{4}(\nu) \epsilon^{4}+O_{\chi}\left(\epsilon^{5},|\nu|\right),
$$

be the fourth-order Taylor expansion, around $\epsilon=0$, of the function $(\epsilon, \nu) \mapsto \mu=\phi(\epsilon, \nu)$. The following statements hold.

(a) For a fixed $(\epsilon, \nu) \in U_{\epsilon}, \epsilon \in \mathbb{R}$ sufficiently small, and $\operatorname{Re}\left(G_{2,1}\left(\xi_{0}\right)\right) \neq 0$, the stability of the periodic orbit of the differential equation (1) is given by the sign of $\operatorname{Re}\left(G_{2,1}\left(\xi_{0}\right)\right)$. When $\operatorname{Re}\left(G_{2,1}\left(\xi_{0}\right)\right)<0$ for $\xi_{0} \in U$, the periodic orbit in the phase portrait of differential equation (1) is stable. As for $\in \in \mathbb{R}$, sufficiently small,

$$
\mu=\phi(\epsilon, \nu)=-\frac{1}{2} \frac{\operatorname{Re}\left(G_{2,1}\left(\xi_{0}\right)\right)}{\partial_{\mu} \gamma\left(\xi_{0}\right)} \epsilon^{2}+O_{\mu}\left(\epsilon^{4},|\nu|\right),
$$


if $\partial_{\mu} \gamma\left(\xi_{0}\right)>0$, the periodic orbit in the phase portrait of (1) exists for $\mu>0$, and if $\partial_{\mu} \gamma\left(\xi_{0}\right)<0$, the periodic orbit in the phase portrait exists for $\mu<0$. If $\operatorname{Re}\left(G_{2,1}\left(\xi_{0}\right)\right)>0$, the periodic orbit in the phase portrait of the differential equation (1) is unstable.

(b) Suppose that for $\xi_{1}=(0,0), \operatorname{Re}\left(G_{2,1}\left(\xi_{1}\right)\right)=0$ and $\operatorname{Re}\left(G_{3,2}\left(\xi_{1}\right)\right) \neq 0$. Then, for $\epsilon \in \mathbb{R}$ sufficiently small, the stability is given by the sign of $\operatorname{Re}\left(G_{3,2}\left(\xi_{1}\right)\right)$. When $\operatorname{Re}\left(G_{3,2}\left(\xi_{1}\right)\right)<0$, the periodic orbit in the phase portrait of the differential equation (1) is stable. As, in this case,

$$
\mu=\phi(\epsilon, \nu)=-\frac{1}{12} \frac{\operatorname{Re}\left(G_{3,2}\left(\xi_{1}\right)\right)}{\partial_{\mu} \gamma\left(\xi_{1}\right)} \epsilon^{4}+O_{\mu}\left(\epsilon^{5},|\nu|\right),
$$

if $\partial_{\mu} \gamma\left(\xi_{1}\right)>0$, the periodic orbit in the phase portrait of the differential equation (1) exists for $\mu>0$, and if $\partial_{\mu} \gamma\left(\xi_{1}\right)<0$, the periodic orbit in the phase portrait of the differential equation (1) exists for $\mu<0$. If $\operatorname{Re}\left(G_{3,2}\left(\xi_{1}\right)\right)>0$, the periodic orbit in the phase portrait of the differential equation (1) is unstable.

Proof. As the sign of the Floquet exponent provides the stability of a periodic orbit, by (109), and (110) the proof is immediate.

Corollary 11 does not deal with the case where $\chi(\epsilon, \nu)=0$ for a set of points $(\epsilon, \nu) \in U_{\epsilon}$. The theory developed up to this point enables us to study the curve of nonhyperbolic periodic orbits $C_{\mathrm{NH}}$ in the parameter plane $(\mu, \nu) \in \mathbb{R}^{2}$, associated with a transversal Hopf point of codimension two. This curve is the set

$$
\chi^{-1}(0)=\left\{(\epsilon, \nu) \in U_{\epsilon}: \chi(\epsilon, \nu)=0\right\}
$$

From the set $\chi^{-1}(0)$ and the Implicit Function Theorem, the parameter $v$ can be obtained as a function of the parameter $\epsilon$. Therefore, the curve $C_{\mathrm{NH}}$ follows from functions $\epsilon \mapsto$ $\nu=\psi(\epsilon)$ and $(\epsilon, \nu) \mapsto \mu=\phi(\epsilon, \nu)$; that is, the curve $C_{\mathrm{NH}}$ can be locally represented as a curve parameterized by $\epsilon$

$$
\Gamma(\epsilon)=(\phi(\epsilon, \psi(\epsilon)), \psi(\epsilon)),
$$

or can be locally represented as the graph of a function

$$
\mu=\Lambda(\nu)
$$

In fact, the Taylor expansion around $\epsilon=0$ of the exponent characteristic is such as in (99), and, therefore,

$$
\chi(\epsilon, \nu)=\epsilon^{2} \Psi(\epsilon, \nu),
$$

where the third-order Taylor expansion around $\epsilon=0$ of the function $(\epsilon, \nu) \mapsto \Psi(\epsilon, \nu)$ is of the following form:

$$
\Psi(\epsilon, \nu)=\frac{1}{2} \chi_{2}(\nu)+\frac{1}{24} \chi_{4}(\nu) \epsilon^{2}+O_{\chi}\left(\epsilon^{3},|\nu|\right) .
$$

It is easy to see that $\Psi^{-1}(0)=\left\{(\epsilon, \nu) \in U_{\epsilon}: \Psi(\epsilon, \nu)=0\right\} \subset$ $\chi^{-1}(0)$. Thus, the study of the curve of nonhyperbolic periodic orbits in the parameter plane $(\mu, \nu) \in \mathbb{R}^{2}$, associated with the differential equation (1), and in the hypotheses of a transversal Hopf bifurcation of codimension two is reduced to the study of the set $\Psi^{-1}(0)$.

The next lemma, whose proof is given in [3], guarantees the existence of the function $\epsilon \mapsto \nu=\psi(\epsilon)$.

Lemma 12. Let

$$
\begin{aligned}
D & : \mathbb{R} \times \mathbb{R}^{4} \longrightarrow \mathbb{R} \\
(x, y) & \longmapsto D(x, y)
\end{aligned}
$$

be a smooth function, where $y=\left(y_{0}, y_{1}, y_{2}, y_{3}\right)$. Suppose that for $\left(0, y^{0}\right) \in \mathbb{R} \times \mathbb{R}^{4}, y^{0}=\left(y_{0}, 0, y_{2}, y_{3}\right)$, the function in (118) satisfies the following assumptions:

(A1) $D\left(0, y^{0}\right)=0$;

(A2) $\partial_{x} D\left(0, y^{0}\right)=0$;

(A3) $\partial_{y_{1}} D\left(0, y^{0}\right) \neq 0$;

(A4) $\partial_{x}^{2} D\left(0, y^{0}\right) \neq 0$.

Then, there exists a unique smooth function

$$
\left(x, y^{0}\right) \longmapsto y_{1}=\phi\left(x, y^{0}\right) \text {, }
$$

such that $y=\Phi\left(x, y^{0}\right)=y^{0}+\left(0, \phi\left(x, y^{0}\right), 0,0\right)$ and $D\left(x, \Phi\left(x, y^{0}\right)\right) \equiv 0$. Moreover, the function $\left(x, y^{0}\right) \mapsto y_{1}=$ $\phi\left(x, y^{0}\right)$ has the following representation:

$$
\phi\left(x, y^{0}\right)=\frac{1}{2 !} \phi_{2}\left(y^{0}\right) x^{2}+O_{\phi}\left(|x|^{3},\left\|y^{0}\right\|\right),
$$

where

$$
\phi_{2}\left(y^{0}\right)=-\frac{\partial_{x}^{2} D\left(0, y^{0}\right)}{\partial_{y_{1}} D\left(0, y^{0}\right)}
$$

The following theorem can be stated now.

Theorem 13. Let $\left(\mathbf{0}, \xi_{1}\right) \in W \times U$ be a transversal Hopf point of codimension two of (1). Then, the curve of nonhyperbolic periodic orbits $C_{N H}$, in the parameter plane $(\mu, \nu) \in \mathbb{R}^{2}$, associated with the differential equation (1), has the following local representations:

$$
\begin{gathered}
\Gamma(\epsilon)=\left(\frac{\operatorname{Re}\left(G_{3,2}\left(\xi_{1}\right)\right)}{12 \partial_{\mu} \gamma\left(\xi_{1}\right)} \epsilon^{4},-\frac{\operatorname{Re}\left(G_{3,2}\left(\xi_{1}\right)\right)}{3 \operatorname{Re}\left(\partial_{\nu} G_{2,1}\left(\xi_{1}\right)\right)} \epsilon^{2}\right)+O_{\Gamma}(\epsilon), \\
\mu=\Lambda(\nu)=\frac{\mu_{2}(\nu)}{\psi_{2}} \nu+\frac{1}{6} \frac{\mu_{4}(\nu)}{\psi_{2}^{2}} \nu^{2}+O_{\Lambda}(|\nu|),
\end{gathered}
$$

where

$$
\psi_{2}=-\frac{\partial_{\epsilon}^{2} \Psi(0,0)}{\partial_{\nu} \Psi(0,0)}=-\frac{2 \operatorname{Re}\left(G_{3,2}\left(\xi_{1}\right)\right)}{3 \partial_{\nu} \operatorname{Re}\left(G_{2,1}\left(\xi_{1}\right)\right)}
$$


Proof. As

$$
\begin{aligned}
& \Psi(0,0)=0, \\
& \partial_{\epsilon} \Psi(0,0)=0, \\
& \partial_{\epsilon}^{2} \Psi(0,0)=\frac{1}{12} \chi_{4}(0)=\frac{2}{3} \operatorname{Re}\left(G_{3,2}\left(\xi_{1}\right)\right) \neq 0, \\
& \partial_{\nu} \Psi(0,0)=\frac{1}{2} \chi_{2}^{\prime}(0)=\partial_{\nu} \operatorname{Re}\left(G_{2,1}\left(\xi_{1}\right)\right) \neq 0 .
\end{aligned}
$$

Lemma 12 guarantees the existence of a smooth function $\epsilon \mapsto$ $\nu=\psi(\epsilon)$ such that $\Psi(\epsilon, \psi(\epsilon)) \equiv 0$, or even, $\chi(\epsilon, \psi(\epsilon)) \equiv 0$. Moreover, the function $\epsilon \mapsto \nu=\psi(\epsilon)$ has the second-order Taylor expansion around $\epsilon=0$ of the following form:

$$
\nu=\psi(\epsilon)=\frac{1}{2 !} \psi_{2} \epsilon^{2}+O_{\psi}\left(\epsilon^{3}\right)
$$

where

$$
\psi_{2}=-\frac{\partial_{\epsilon}^{2} \Psi(0,0)}{\partial_{\nu} \Psi(0,0)}=-\frac{2 \operatorname{Re}\left(G_{3,2}\left(\xi_{1}\right)\right)}{3 \partial_{\nu} \operatorname{Re}\left(G_{2,1}\left(\xi_{1}\right)\right)}
$$

Thus,

$$
\nu=\psi(\epsilon)=-\frac{\operatorname{Re}\left(G_{3,2}\left(\xi_{1}\right)\right)}{3 \partial_{\nu} \operatorname{Re}\left(G_{2,1}\left(\xi_{1}\right)\right)} \epsilon^{2}+O_{\psi}\left(\epsilon^{3}\right),
$$

and substituting (128) into the function $(\epsilon, \nu) \mapsto \mu=\phi(\epsilon, \nu)$ results in the following Taylor expansion:

$$
\mu=\phi(\epsilon, \psi(\epsilon))=\frac{\operatorname{Re}\left(G_{3,2}\left(\xi_{1}\right)\right)}{12 \partial_{\mu} \gamma\left(\xi_{1}\right)} \epsilon^{4}+O_{\phi}\left(\epsilon^{5}\right) .
$$

So, there is a curve in the parameter plane, $\epsilon \mapsto \Gamma(\epsilon)=$ $(\phi(\epsilon, \psi(\epsilon)), \psi(\epsilon))$, that can be parameterized by $\epsilon$ and represented as in (122). Another representation for this curve is obtained when the Implicit Function Theorem is applied to the following function:

$$
\epsilon^{2}=\frac{2}{\psi_{2}} \nu+O_{\epsilon}\left(|\nu|^{2}\right)
$$

By substituting (130) into (110), the curve $\epsilon \mapsto \Gamma(\epsilon)$ can also be represented locally as

$$
\begin{aligned}
\mu=\Lambda(\nu) & =\frac{1}{2} \mu_{2}(\nu)\left(\frac{2}{\psi_{2}} v\right)+\frac{1}{24} \mu_{4}(v)\left(\frac{2}{\psi_{2}} v\right)^{2}+O_{\Lambda}(|v|) \\
& =\frac{\mu_{2}(\nu)}{\psi_{2}} \nu+\frac{1}{6} \frac{\mu_{4}(\nu)}{\psi_{2}^{2}} v^{2}+O_{\Lambda}(|\nu|) .
\end{aligned}
$$

Therefore, there exists a curve $\Gamma$ in the parameter plane that locally has the representation (122) or (123). By the hypotheses of the transversal Hopf bifurcation of codimension two, $\operatorname{Re}\left(G_{3,2}\left(\xi_{1}\right)\right) \neq 0$ and equation $z^{\prime}=g(z, \bar{z}, \xi)$ are locally topologically equivalent, around $z=0$, to the complex differential equation (67). Therefore, the curve of nonhyperbolic periodic orbits has the representation (122) or (123).
Example 14. For the complex differential equation (67), we have

$$
\begin{gathered}
\gamma(\alpha, \beta)=\alpha, \\
\eta(\alpha, \beta)=1, \\
G_{2,1}(\alpha, \beta)=2 \beta, \\
G_{3,2}(\alpha, \beta)=12 s .
\end{gathered}
$$

So, by Theorem 13, the curve of nonhyperbolic periodic orbits has the following representations:

$$
\begin{aligned}
& \Gamma(\epsilon)=\left(s \epsilon^{4},-2 s \epsilon^{2}\right)+O_{\Gamma}(\epsilon), \\
& \alpha=\Lambda(\beta)=\frac{1}{4 s} \beta^{2}+O_{\Lambda}(\beta),
\end{aligned}
$$

which agree with (68) and (69), respectively.

The local representations (122) and (123) in Theorem 13 are valid when the Hopf curve is the set $\{(\mu, \nu): \mu=0\}$. If the Hopf curve is the set $\{(\mu, \nu): \mu=\varphi(\nu)\}$ and the transversal Hopf bifurcation of codimension two occurs for $\xi_{1}=\left(\mu_{1}, \nu_{1}\right) \neq(0,0)$, it is easy to show that the local representations are given by

$$
\begin{gathered}
\Gamma(\epsilon)=\left(\mu_{1}+\frac{1}{2} \varphi_{2} \epsilon^{2}+\frac{\operatorname{Re}\left(G_{3,2}\left(\xi_{1}\right)\right)}{12 \partial_{\mu} \gamma\left(\xi_{1}\right)} \epsilon^{4},\right. \\
\left.\nu_{1}-\frac{\operatorname{Re}\left(G_{3,2}\left(\xi_{1}\right)\right)}{3 \operatorname{Re}\left(\partial_{\nu} G_{2,1}\left(\xi_{1}\right)\right)} \epsilon^{2}\right)+O_{\Gamma}(\epsilon), \\
\mu=\Lambda(\nu)=\varphi(\nu)+\frac{\mu_{2}(\nu)}{\psi_{2}}\left(\nu-\nu_{1}\right) \\
+\frac{1}{6} \frac{\mu_{4}(\nu)}{\psi_{2}^{2}}\left(\nu-\nu_{1}\right)^{2}+O_{\Lambda}(|\nu|),
\end{gathered}
$$

for $v \leq v_{1}$, where

$$
\mu_{1}=\varphi\left(\nu_{1}\right), \quad \varphi_{2}=\psi_{2} \partial_{\nu} \varphi\left(\nu_{1}\right)
$$

The next two sections present applications of the theory developed here in an extension of the van der Pol equation known as the Liénard equation and in Bazykin's predatorprey system and show how local representations of the the curve $C_{\mathrm{NH}}$ are obtained.

\section{Liénard Equation}

One of the pioneers in nonlinear electrical circuits was, undoubtedly, Balthasar van der Pol, through studies with triodes (vacuum tubes). Balthasar van der Pol showed that in circuits with triodes, the electrical quantities can exhibit nonlinear oscillations under certain conditions. Nowadays, it is known that the model of this circuit with triode presents a Hopf bifurcation. In a simple and theoretical way, the electric circuit of van der Pol consists of a triode, a capacitor of capacitance $C$, and an inductor of inductance $L$, according to the diagram of Figure 1. 


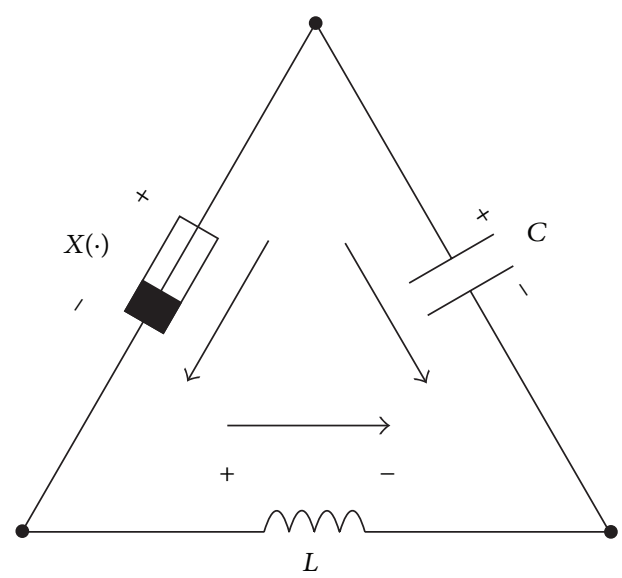

Figure 1: van der Pol circuit diagram.

Let $v_{C}, i_{C}$ and $v_{L}, i_{L}$ be the models of voltage and current in the capacitor and inductor, respectively. The triode of van der Pol, by the hypothesis, satisfies the generalized Ohm's law $i_{R} \mapsto v_{R}=X\left(i_{R}\right)$, where $v_{R}$ and $i_{R}$ are the models of voltage and current of the triode of van der Pol, respectively. Applying Kirchhoff's laws to the van der Pol electrical circuit model and using the capacitor and inductor equations, it follows that

$$
\begin{aligned}
& i_{R}=i_{L}=-i_{C}, \quad v_{r}+v_{L}-v_{C}=0, \\
& v_{L}=L \frac{d}{d t} i_{L}, \quad i_{C}=C \frac{d}{d t} v_{C} .
\end{aligned}
$$

Therefore, the van der Pol circuit model is of the following form:

$$
\begin{aligned}
& L \frac{d}{d t} i_{L}=v_{c}-X\left(i_{L}\right), \\
& C \frac{d}{d t} v_{c}=-i_{L} .
\end{aligned}
$$

The study of differential equation (137) is simplified by the change of coordinates and time

$$
x=\frac{L}{\sqrt{L C}} i_{L}, \quad y=v_{C}, \quad \tau=\frac{1}{\sqrt{L C}} t,
$$

which leads to the differential equation

$$
\begin{gathered}
x^{\prime}=\frac{d}{d \tau} x=y-x(x), \\
y^{\prime}=\frac{d}{d \tau} y=-x,
\end{gathered}
$$

where $\mathscr{X}(x)=X((C / \sqrt{L C}) x)$. Suppose that

$$
x \longmapsto X(x)=-\mu x+\nu x^{3}+\frac{1}{5} x^{5} .
$$

In the literature, the differential equation (139) satisfying (140) is known as the Liénard-type equation.
The Liénard equation has a unique equilibrium point $(\mathbf{0}, \xi) \in \mathbb{R}^{2} \times \mathbb{R}^{2}$, with $\xi=(\mu, \nu)$, and the linear part of the vector field, evaluated at $(\mathbf{0}, \xi)$,

$$
A(\xi)=\left(\begin{array}{cc}
\mu & 1 \\
-1 & 0
\end{array}\right)
$$

has eigenvalues $\lambda$ and $\bar{\lambda}$, with

$$
\lambda(\xi)=\gamma(\xi)+i \eta(\xi)=\frac{1}{2} \mu+i \frac{1}{2} \sqrt{4-\mu^{2}},
$$

for $\mu \in(-2,2)$. When $\xi=\xi_{0}=(0, \nu), \gamma\left(\xi_{0}\right)=0$, and $\eta\left(\xi_{0}\right)=$ 1 , which indicates the occurrence of Hopf bifurcations. The eigenvectors $q\left(\xi_{0}\right) \in \mathbb{C}^{2}$ and $p\left(\xi_{0}\right) \in \mathbb{C}^{2}$, where $q\left(\xi_{0}\right)$ is normalized with respect to $p\left(\xi_{0}\right)$ according to (8), are chosen as

$$
q\left(\xi_{0}\right)=\left(-\frac{i}{2}, \frac{1}{2}\right), \quad p\left(\xi_{0}\right)=(-i, 1) .
$$

In the case of the Liénard equation, the symmetric multilinear functions are given by

$$
\begin{aligned}
& B(\mathbf{x}, \mathbf{y}, \xi)=(0,0), \\
& C(\mathbf{x}, \mathbf{y}, \mathbf{u}, \xi)=\left(6 v x_{1} y_{1} u_{1}, 0\right), \\
& D(\mathbf{x}, \mathbf{y}, \mathbf{u}, \mathbf{v}, \xi)=(0,0), \\
& E(\mathbf{x}, \mathbf{y}, \mathbf{u}, \mathbf{v}, \mathbf{w}, \xi)=\left(24 x_{1} y_{1} u_{1} v_{1} w_{1}, 0\right) .
\end{aligned}
$$

Thus, for $k=2,3, \ldots$ and $j=0,1, \ldots, k$, the only nonzero coefficients $g_{k-j, j}(\xi)$ are

$$
\begin{aligned}
g_{3,0}\left(\xi_{0}\right) & =g_{1,2}\left(\xi_{0}\right)=-g_{2,1}\left(\xi_{0}\right)=-g_{0,3}\left(\xi_{0}\right)=\frac{3}{4} \nu, \\
g_{4,1}\left(\xi_{0}\right) & =g_{2,3}\left(\xi_{0}\right)=g_{0,5}\left(\xi_{0}\right)=-g_{5,0}\left(\xi_{0}\right) \\
& =-g_{3,2}\left(\xi_{0}\right)=-g_{1,4}\left(\xi_{0}\right)=\frac{3}{4} .
\end{aligned}
$$

The eigenvectors $\partial_{\mu} q\left(\xi_{0}\right)$ and $\partial_{\mu} p\left(\xi_{0}\right)$ and the coefficients $\partial_{\mu} g_{2,0}\left(\xi_{0}\right), \partial_{\mu} g_{1,1}\left(\xi_{0}\right), \partial_{\mu} g_{0,2}\left(\xi_{0}\right)$, and $\partial_{\mu} g_{2,1}\left(\xi_{0}\right)$, computed by Propositions 7 and 8 , are such that

$$
\begin{aligned}
& \partial_{\mu} q\left(\xi_{0}\right)=\left(-\frac{1}{8}, \frac{i}{8}\right), \\
& \partial_{\mu} p\left(\xi_{0}\right)=\left(\frac{1}{4},-\frac{i}{4}\right), \\
& \partial_{\mu} g_{2,0}\left(\xi_{0}\right)=\partial_{\mu} g_{1,2}\left(\xi_{0}\right)=\partial_{\mu} g_{0,2}\left(\xi_{0}\right)=0, \\
& \partial_{\mu} g_{2,1}\left(\xi_{0}\right)=\frac{3}{8} i \nu .
\end{aligned}
$$

Thus, from the previous results and by (50), (63), and (64), it follows that

$$
\begin{gathered}
G_{2,1}\left(\xi_{0}\right)=-\frac{3}{4} \nu, \quad \partial_{\mu} G_{2,1}\left(\xi_{0}\right)=\frac{3}{8} i \nu, \\
G_{3,2}\left(\xi_{0}\right)=-\frac{3}{4}-\frac{81}{64} i \nu^{2} .
\end{gathered}
$$


Therefore, the first Lyapunov coefficient is given by

$$
l_{1}\left(\xi_{0}\right)=\operatorname{Re}\left(G_{2,1}\left(\xi_{0}\right)\right)=-\frac{3}{4} \nu,
$$

and since

$$
\partial_{\mu} \gamma\left(\xi_{0}\right)=\frac{1}{2}
$$

the Liénard equation presents a transversal Hopf bifurcation of codimension one for $\mu=0$ and $\nu \neq 0$. From Corollary 11,

$$
\begin{gathered}
\mu=\left(\frac{3}{4} \nu\right) \epsilon^{2}+O_{\mu}\left(\epsilon^{4},|\nu|\right), \\
\chi(\epsilon, \nu)=\left(-\frac{3}{4} \nu\right) \epsilon^{2}+O_{\chi}\left(\epsilon^{3},|\nu|\right),
\end{gathered}
$$

and if $\nu<0$, then there exists a unique unstable periodic orbit in the phase portrait of the Liénard equation when $\mu<0$, and if $\nu>0$, the periodic orbit is stable and there exists for $\mu>0$.

For $v=0$, the Liénard equation has a transversal Hopf point of codimension two, and the second Lyapunov coefficient is given by

$$
l_{2}\left(\xi_{1}\right)=\operatorname{Re}\left(G_{3,2}\left(\xi_{1}\right)\right)=-\frac{1}{16},
$$

where $\xi_{1}=(0,0)$. Since

$$
\partial_{\nu} \operatorname{Re}\left(G_{2,1}\left(\xi_{1}\right)\right) \partial_{\mu} \gamma\left(\xi_{1}\right)=\left(-\frac{3}{4}\right)\left(\frac{1}{2}\right)=-\frac{3}{8} \neq 0,
$$

by Corollary 11 and Theorem 13, the Liénard equation has a bifurcation diagram as shown in Figure 2.

The curve of nonhyperbolic periodic orbits has the following local representations:

$$
\Gamma(\epsilon)=(\mu(\epsilon, \nu(\epsilon)), \nu(\epsilon))=\left(-\frac{1}{8} \epsilon^{4},-\frac{1}{3} \epsilon^{2}\right)+O_{\Gamma}(\epsilon),
$$

as a curve parameterized by $\epsilon$ or as the graph of the function

$$
\mu=\Lambda(\nu)=-\frac{9}{8} v^{2}+O_{v}\left(v^{3}\right)
$$

for $v \leq 0$.

Figure 3 emphasizes the comparison between the curve of nonhyperbolic periodic orbits $C_{\mathrm{NH}}$ of (139) obtained numerically with the software MATCONT (see [5]) and the quadratic approximation (154).

\section{Bazykin's Predator-Prey System}

Consider the dynamics of a predator-prey ecosystem, whose model is

$$
\begin{gathered}
x^{\prime}=\frac{x^{2}(1-x)}{\mu+x}-x y, \\
y^{\prime}=-\gamma y(\nu-x),
\end{gathered}
$$

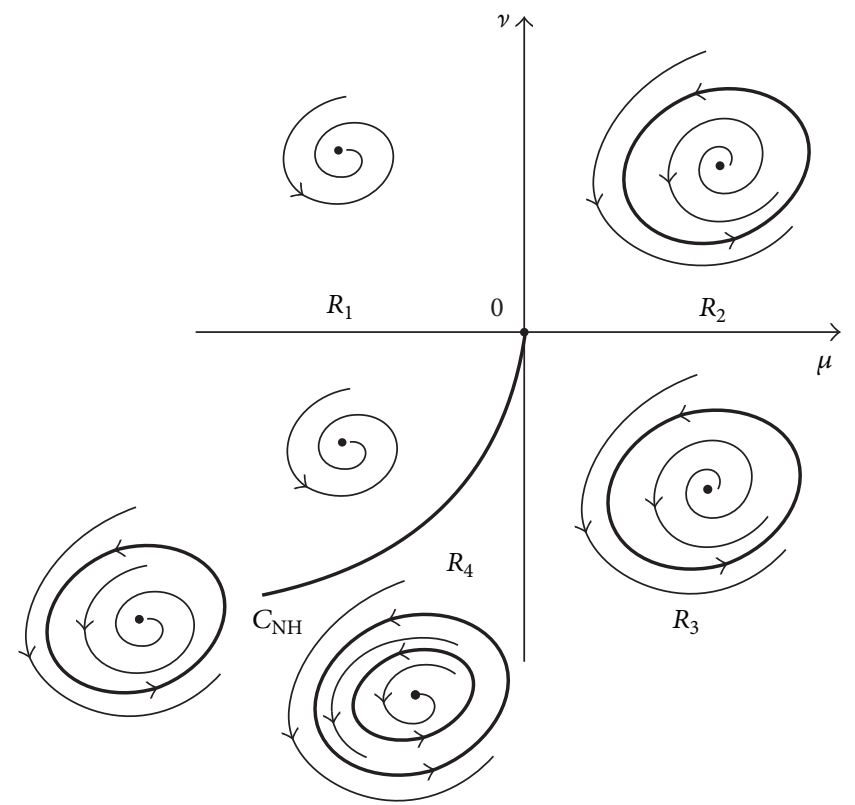

FIGURE 2: Bifurcation diagram of the Liénard equation (139).

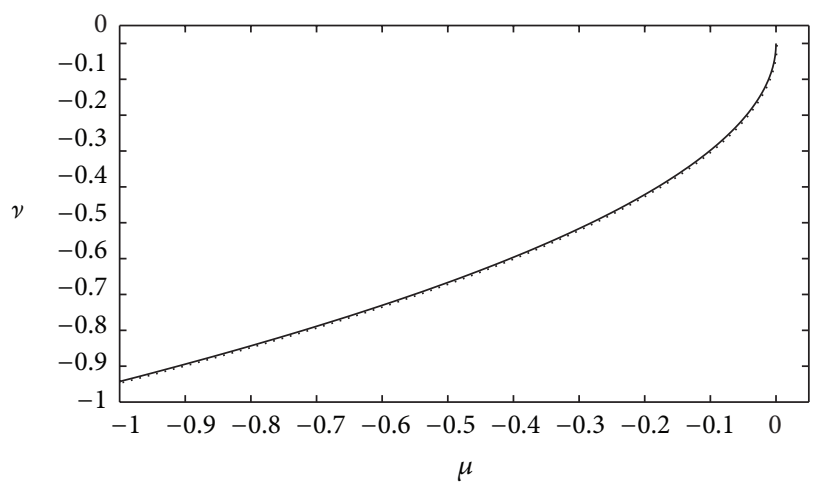

FIGURE 3: Comparison between the curve of nonhyperbolic periodic orbits $C_{\mathrm{NH}}$ of (139): the dotted curve was obtained numerically with the software MATCONT and the continuous curve from the representation (154).

where $\gamma>0$ (fixed), $\mu \geq 0$, and $0 \leq \nu<1$ are parameters. Model (155) is known in the literature as Bazykin's predatorprey system. See [1] or [10].

Taking $\gamma=1$, the equilibrium point of interest is

$$
\left(\mathbf{x}_{0}(\xi), \xi\right)=\left(\left(\nu, \frac{\nu(1-\nu)}{\mu+\nu}\right), \xi\right) .
$$

For

$$
\mu=\varphi(\nu)=\frac{v^{2}}{1-2 v},
$$

the linear part of the vector field, evaluated at $\left(\mathbf{x}_{\mathbf{0}}(\xi), \xi\right)$,

$$
A(\xi)=\left(\begin{array}{cc}
\frac{\nu\left(\mu(1-2 \nu)-\nu^{2}\right)}{(\mu+\nu)^{2}} & -v \\
\frac{\nu(1-\nu)}{\mu+\nu} & 0
\end{array}\right),
$$


has eigenvalues $\lambda$ and $\bar{\lambda}$, where $\lambda\left(\xi_{0}\right)=i \omega_{0}(\nu), \omega_{0}(\nu)=$ $\sqrt{\nu(1-2 \nu)}$ and $\xi_{0}=(\varphi(\nu), \nu)$.

The eigenvectors $q\left(\xi_{0}\right) \in \mathbb{C}^{2}$ and $p\left(\xi_{0}\right) \in \mathbb{C}^{2}$ are chosen as

$$
q\left(\xi_{0}\right)=\left(\frac{i \nu}{2 \omega_{0}(\nu)}, \frac{1}{2}\right), \quad p\left(\xi_{0}\right)=\left(\frac{i \omega_{0}(\nu)}{\nu}, 1\right)
$$

and by Proposition 7,

$$
\begin{aligned}
& \partial_{\mu} \gamma\left(\xi_{0}\right)=\frac{(1-2 \nu)^{3}}{2 \nu(1-\nu)^{2}}, \\
& \partial_{\mu} \eta\left(\xi_{0}\right)=-\frac{(1-2 \nu)^{2}}{2(1-\nu) \omega_{0}(\nu)}, \\
& \partial_{\mu}^{2} \gamma\left(\xi_{0}\right)=-\frac{2(1-2 \nu)^{4}}{\nu^{2}(1-\nu)^{3}}, \\
& \partial_{\mu} q\left(\xi_{0}\right)=\left(\frac{1-(1-\nu)\left(4 \nu-i \omega_{0}(\nu)\right)}{8 \nu(1-v)^{2}},\right. \\
& \left.\frac{\omega_{0}(\nu)^{2}\left(i \omega_{0}(\nu)^{3}-v^{2}(1-v)\right)}{8 v^{4}(1-\nu)^{2}}\right), \\
& \partial_{\mu} p\left(\xi_{0}\right)=\left(\frac{i \omega_{0}(\nu)^{3}\left(i \omega_{0}(\nu)^{3}-\nu^{2}(1-\nu)\right)}{4 v^{5}(1-v)^{2}}\right. \\
& \left.-\frac{\omega_{0}(\nu)^{2}\left(i \omega_{0}(\nu)^{3}-v^{2}(1-\nu)\right)}{4 \nu^{4}(1-\nu)^{2}}\right) .
\end{aligned}
$$

The symmetric multilinear functions are given by

$$
\begin{aligned}
B(\mathbf{x}, \mathbf{y}, \xi)=( & -x_{2} y_{1}-\frac{2\left(v^{3}+3 \mu \nu^{2}+\mu^{2}(3 v-1)\right)}{(\mu+\nu)^{3}} x_{1} y_{1} \\
& \left.-x_{1} y_{2}, x_{2} y_{1}+x_{1} y_{2}\right)
\end{aligned}
$$$$
\partial_{\mu} B(\mathbf{x}, \mathbf{y}, \xi)=\left(\frac{2 \mu(2 v+\mu(3 v-1))}{(\mu+v)^{4}} x_{1} y_{1}, 0\right) \text {, }
$$$$
C(\mathbf{x}, \mathbf{y}, \mathbf{u}, \xi)=\left(-\frac{6 \mu^{2}(\mu+1)}{(\mu+\nu)^{4}} x_{1} y_{1} z_{1}, 0\right) \text {, }
$$$$
\partial_{\mu} C(\mathbf{x}, \mathbf{y}, \mathbf{u}, \xi)=\left(\frac{6 \mu\left(\mu^{2}+(2-3 \nu) \mu-2 \nu\right)}{(\mu+\nu)^{5}} x_{1} y_{1} z_{1}, 0\right) \text {, }
$$$$
D(\mathbf{x}, \mathbf{y}, \mathbf{u}, \mathbf{v}, \xi)=\left(\frac{24 \mu^{2}(\mu+1)}{(\mu+v)^{5}} x_{1} y_{1} z_{1} u_{1}, 0\right) \text {, }
$$$$
E(\mathbf{x}, \mathbf{y}, \mathbf{u}, \mathbf{v}, \mathbf{w}, \xi)=\left(-\frac{120 \mu^{2}(\mu+1)}{(\mu+\nu)^{6}} x_{1} y_{1} z_{1} u_{1} v_{1}, 0\right) \text {. }
$$

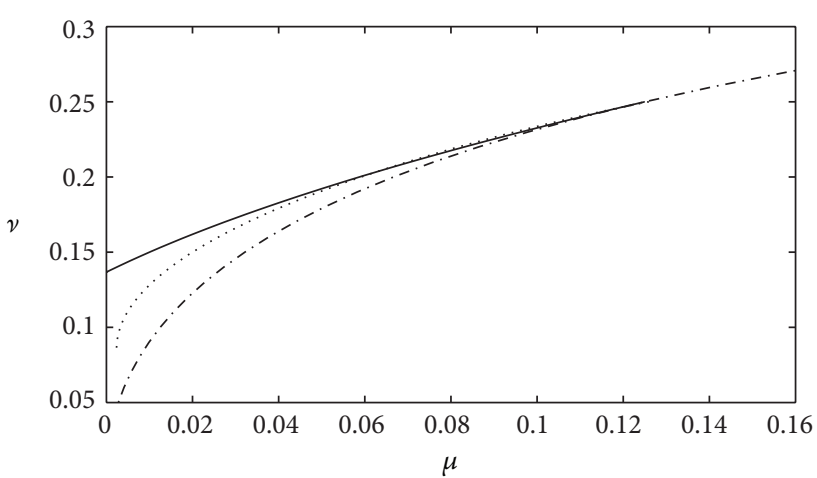

FIGURE 4: Comparison between the curve of nonhyperbolic periodic orbits $C_{\mathrm{NH}}$ of (155): the dotted curve was obtained numerically with the software MATCONT and the continuous curve from the representation (164). The dot-dashed curve is the Hopf curve.

Therefore, from the previous results,

$$
\begin{gathered}
\operatorname{Re}\left(G_{2,1}\left(\xi_{0}\right)\right)=\frac{1-4 \nu}{4(1-\nu)^{2}}, \\
\partial_{\mu} \operatorname{Re}\left(G_{2,1}\left(\xi_{0}\right)\right)=-\frac{5(1-2 \nu)\left(40 \nu^{3}-67 v^{2}+27 \nu-2\right)}{72 \nu^{2}(1-v)^{4}}, \\
\partial_{\nu} \operatorname{Re}\left(G_{2,1}\left(\xi_{0}\right)\right)=-\frac{2 \nu+1}{2(1-\nu)^{3}}, \\
\operatorname{Re}\left(G_{3,2}\left(\xi_{0}\right)\right)=\frac{292 \nu^{4}-485 v^{3}+286 \nu^{2}-68 v+5}{24 \nu(1-2 \nu)^{2}(1-v)^{4}} .
\end{gathered}
$$

When $\nu=\nu_{1}=1 / 4, \mu_{1}=\varphi\left(\nu_{1}\right)=1 / 8$. Thus, for $\xi_{1}=$ $\left(\mu_{1}, \nu_{1}\right)=(1 / 8,1 / 4)$, Bazykin's system (155) has a transversal Hopf point of codimension two, since $\operatorname{Re}\left(G_{2,1}\left(\xi_{1}\right)\right)=0$, $\operatorname{Re}\left(G_{3,2}\left(\xi_{1}\right)\right)=-32 / 27$, and

$$
\partial_{\nu} \operatorname{Re}\left(G_{2,1}\left(\xi_{1}\right)\right) \partial_{\mu} \gamma\left(\xi_{1}\right)=\left(-\frac{16}{9}\right)\left(\frac{4}{9}\right)=-\frac{64}{81} \neq 0 .
$$

Using (134), the curve of nonhyperbolic periodic orbits has the following local representations:

$$
\Gamma(\epsilon)=\left(\frac{1}{8}-\frac{\epsilon^{2}}{3}+\frac{14 \epsilon^{4}}{81}, \frac{1}{4}-\frac{2 \epsilon^{2}}{9}\right)+O_{\Gamma}(\epsilon),
$$

as a curve parameterized by $\epsilon$ or as a graph of the function

$$
\begin{aligned}
\mu= & \Lambda(\nu)=\frac{v^{2}}{1-2 v}+\frac{9 v(1-4 v)^{2}}{32(2 v-1)^{3}} \\
& +\frac{27(4 v-1)^{2} v\left(120 v^{3}-98 v^{2}+7 v+1\right)}{2048(v-1)^{2}(2 v-1)^{5}} \\
& +O_{\nu}\left(v^{3}\right)
\end{aligned}
$$

for $v \leq v_{1}=1 / 4$.

Figure 4 emphasizes the comparison between the curve of nonhyperbolic periodic orbits $C_{\mathrm{NH}}$ of (155) obtained 


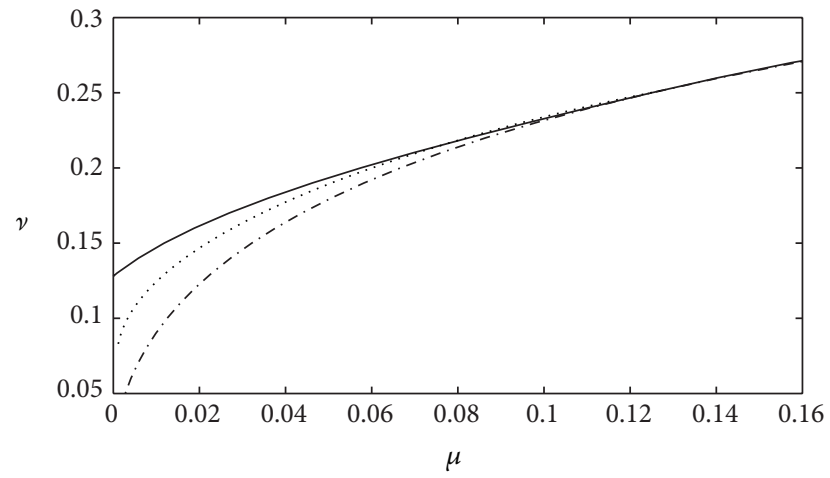

FIGURE 5: Comparison between the curve of nonhyperbolic periodic orbits $C_{\mathrm{NH}}$ of (155): the dotted curve was obtained numerically with the software MATCONT and the continuous curve from the representation (165). The dot-dashed curve is the Hopf curve.

numerically with the software MATCONT and the quadratic approximation (164).

The comparison between the curve of nonhyperbolic periodic orbits $C_{\mathrm{NH}}$ of (155) obtained numerically with the software MATCONT and the approximation (165) is shown in Figure 5.

\section{Concluding Comments}

This paper shows how to obtain approximations of periodic orbits of a family of differential equations in the plane that has a transversal Hopf point. Moreover, if the family of differential equations has a transversal Hopf point of codimension two, then it is also possible to build an approximation to the curve of nonhyperbolic periodic orbits in the bifurcation diagram. These results are summarized in Corollary 11 and Theorem 13. Example 14, the study of the Liénard equation (139) in Section 4, and Bazykin's predator-prey system in Section 5 demonstrate the applicability of the theory. See also Figures 3, 4, and 5.

Although the theory is formulated for a family of differential equations in the plane, it can be applied to any family of differential equations in $\mathbb{R}^{n}$ that presents a transversal Hopf bifurcation of codimension two. For this, it is necessary to use the Center Manifold Theorem, or more precisely, to apply the proposed theory to the family of differential equations in $\mathbb{R}^{n}$ restricted to the center manifold.

\section{Acknowledgments}

The second author is partially supported by CNPq Grant 301758/2012-3 and by FAPEMIG Grant PPM-0092-13. The third author thanks CNPq and INERGE for partially supporting this paper. This work was initiated when the second author visited the Laboratoire de Mathématiques, Informatique et Applications of Université de Haute Alsace (2011).

\section{References}

[1] Y. A. Kuznetsov, Elements of Applied Bifurcation Theory, Springer, New York, NY, USA, 2nd edition, 1998.
[2] Yu. A. Kuznetsov, "Numerical normalization techniques for all codim 2 bifurcations of equilibria in ODE's," SIAM Journal on Numerical Analysis, vol. 36, no. 4, pp. 1104-1124, 1999.

[3] D. C. Braga, L. F. Mello, C. Rocşoreanu, and M. Sterpu, "Controllable Hopf bifurcations of codimensions one and two in linear control systems," International Journal of Bifurcation and Chaos in Applied Sciences and Engineering, vol. 21, no. 9, pp. 2665-2678, 2011.

[4] W.-J. Beyn, A. Champneys, E. Doedel, W. Govaerts, Y. A. Kuznetsov, and B. Sandstede, "Numerical continuation, and computation of normal forms," in Handbook of Dynamical Systems, Vol. 2, chapter 4, pp.149-219, North-Holland, Amsterdam, The Netherlands, 2002.

[5] A. Dhooge, W. Govaerts, and Yu. A. Kuznetsov, "MATCONT: a MATLAB package for numerical bifurcation analysis of ODEs," Association for Computing Machinery. Transactions on Mathematical Software, vol. 29, no. 2, pp. 141-164, 2003.

[6] G. Iooss and D. Joseph, Elementary Stability and Bifurcation Theory, Springer, New York, NY, USA, 2nd edition, 2004.

[7] Yu. A. Kuznetsov, H. G. E. Meijer, W. Govaerts, and B. Sautois, "Switching to nonhyperbolic cycles from codim 2 bifurcations of equilibria in ODEs," Physica D, vol. 237, no. 23, pp. 3061-3068, 2008.

[8] J. Sotomayor, L. F. Mello, and D. C. Braga, "Bifurcation analysis of the Watt governor system," Computational \& Applied Mathematics, vol. 26, no. 1, pp. 19-44, 2007.

[9] C. Chicone, Ordinary Differential Equations with Applications, Springer, New York, NY, USA, 2006.

[10] A. D. Bazykin and A. I. Khibnik, "On sharp excitation of selfoscillations in a Volterra-type model," Biophysika, vol. 26, pp. 851-853, 1981 (Russian). 


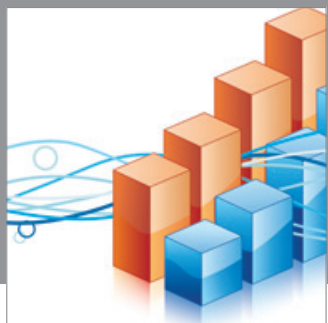

Advances in

Operations Research

mansans

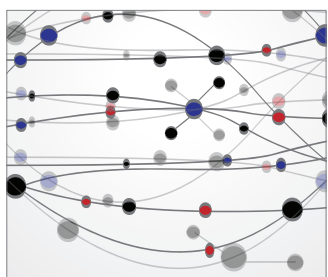

The Scientific World Journal
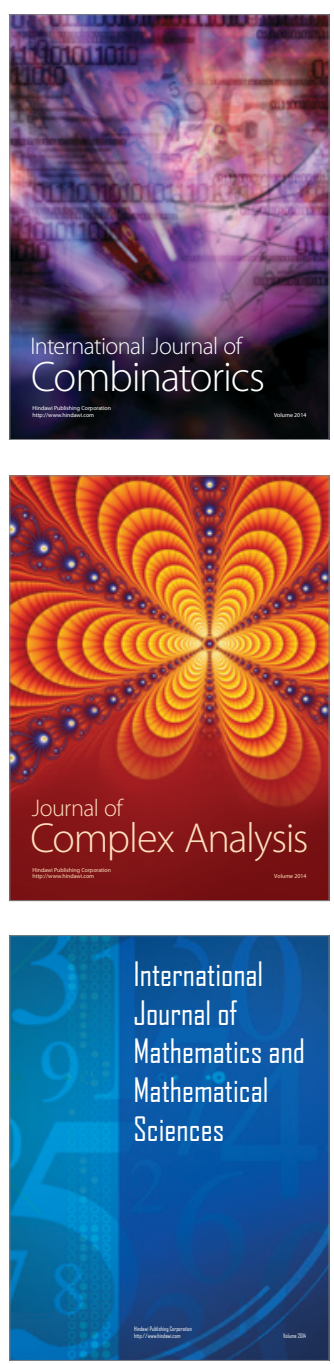
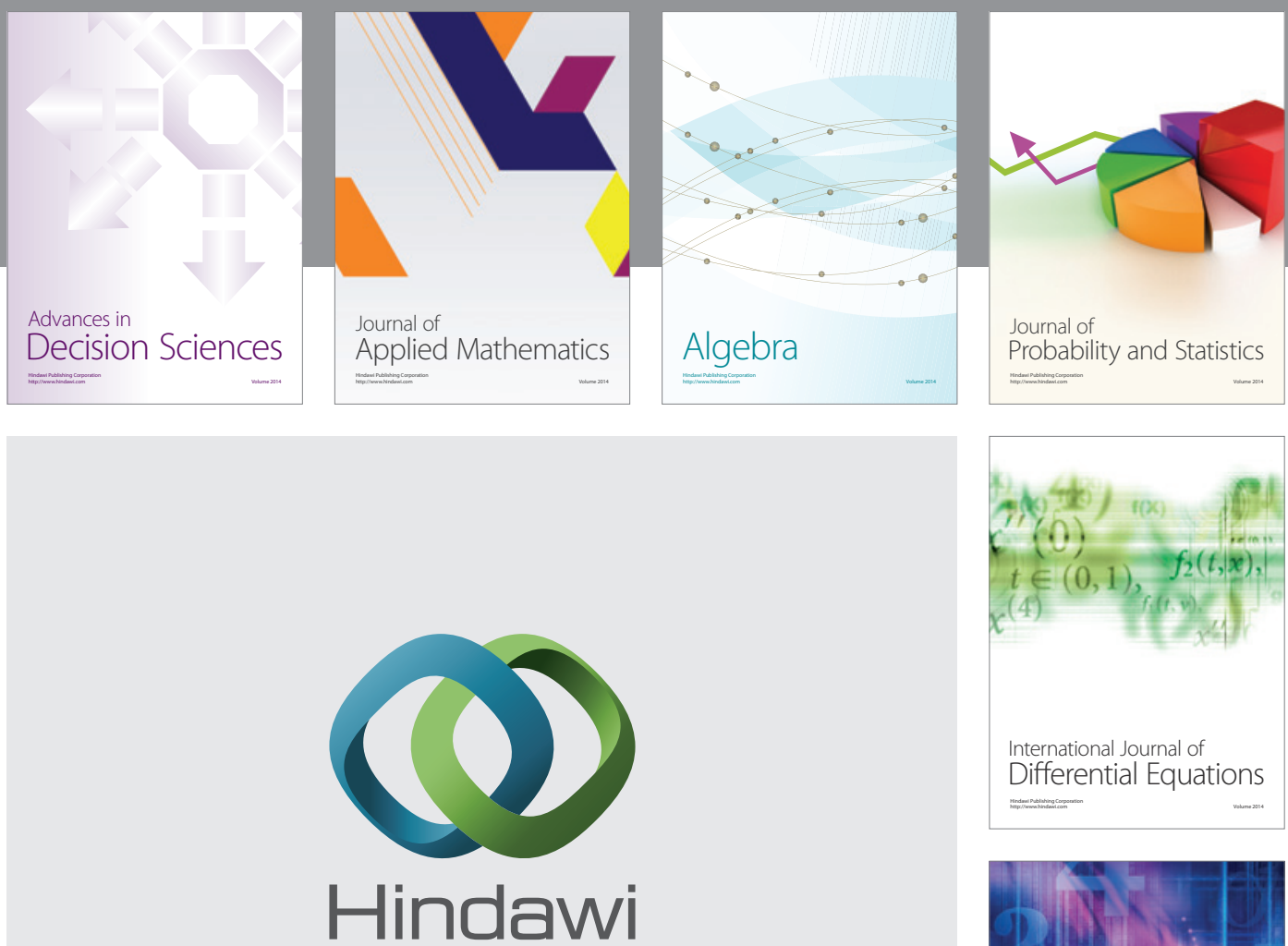

Submit your manuscripts at http://www.hindawi.com
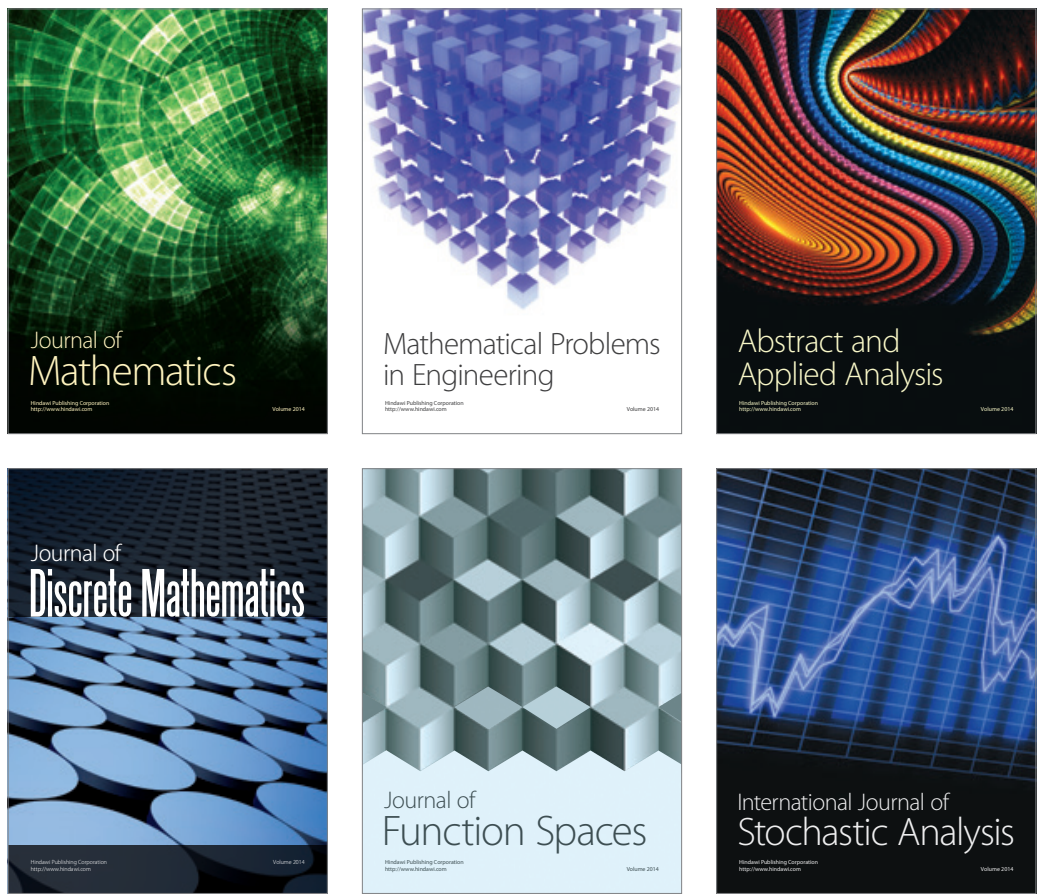

Journal of

Function Spaces

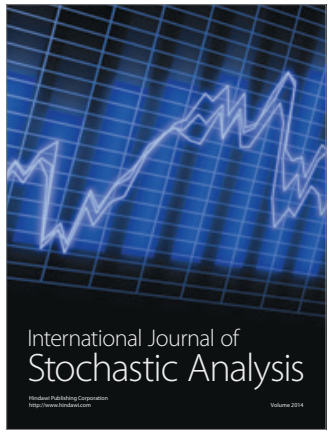

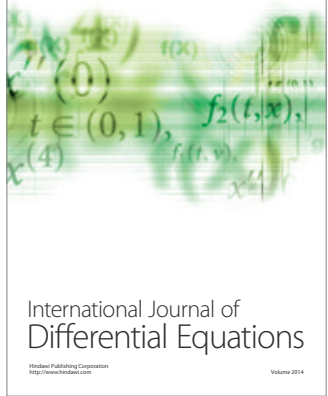
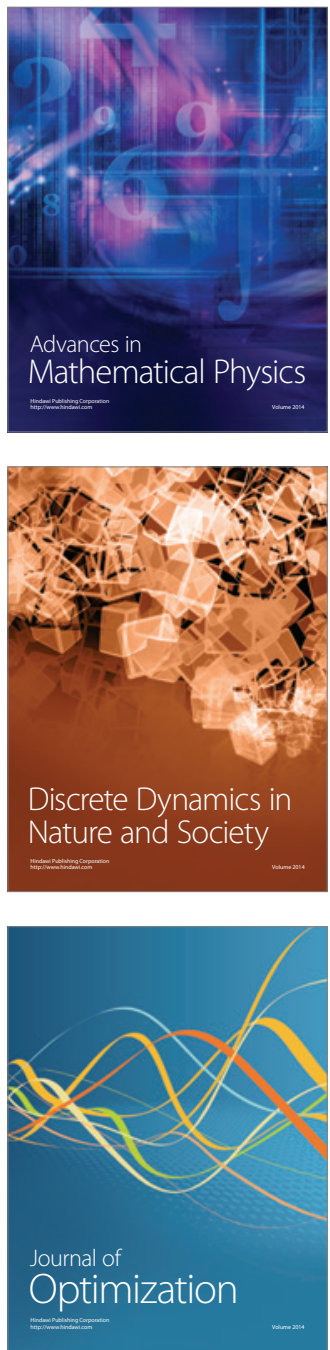